\title{
Transverse Meissner physics of planar superconductors with columnar pins
}

\author{
Gil Refael, ${ }^{1}$ Walter Hofstetter, ${ }^{2}$ and David R. Nelson ${ }^{3}$ \\ ${ }^{1}$ Department of Physics, California Institute of Technology, MC 114-36, Pasadena, California 91125, USA \\ ${ }^{2}$ Institut für Theoretische Physik, Johann Wolfgang Goethe-Universität, 60438 Frankfurt am Main, Germany \\ ${ }^{3}$ Department of Physics, Harvard University, Cambridge, Massachusetts 02138, USA
}

(Received 5 June 2006; revised manuscript received 9 October 2006; published 29 November 2006)

\begin{abstract}
The statistical mechanics of thermally excited vortex lines with columnar defects can be mapped onto the physics of interacting quantum particles with quenched random disorder in one less dimension. The destruction of the Bose glass phase in type-II superconductors, when the external magnetic field is tilted sufficiently far from the column direction, is described by a poorly understood non-Hermitian quantum phase transition. We present here exact results for this transition in $(1+1)$ dimensions, obtained by mapping the problem in the hard core limit onto one-dimensional fermions described by a non-Hermitian tight binding model. Both site randomness and the relatively unexplored case of bond randomness are considered. Analysis near the mobility edge and near the band center in the latter case is facilitated by a real space renormalization group procedure used previously for Hermitian quantum problems with quenched randomness in one dimension.
\end{abstract}

DOI: 10.1103/PhysRevB.74.174520

PACS number(s): 74.78. $-\mathrm{w}, 72.15 . \mathrm{Rn}, 74.40 .+\mathrm{k}$

\section{INTRODUCTION}

The physical properties of vortices in random pinning potentials have been the focus of investigation for many years. In addition to presenting a challenging problem for statistical-mechanics analysis, ${ }^{1,2}$ the understanding of vortex pinning is crucial for the use of superconducting magnets, as well as for other applications.

This paper focuses on a particular aspect of this problem: Vortices trapped inside a slab of superconducting material with columnar defects. Vortices can minimize their core energy by occupying such defects, particularly if the applied magnetic field and the defects are parallel. If, instead, a field at a certain angle to the defect direction is applied, the energetics of vortices penetrating the superconductor will exhibit a competition between the attractive columnar-defect potential, and the energy cost of the superconductor's magnetization being tilted relative to the applied field. At low tilts of the applied field relative to an irregular array of parallel columnar defects, vortices remain localized along the defects: They exhibit a transverse Meissner effect. At a critical value of the tilt, however, vortices may undergo a delocalization transition, and a breakdown of the transverse-Meissner effect. Figure 1 depicts a generic phase diagram of a vortex system on a lattice of random columnar defects.

In general, the problem of vortex pinning in a superconductor with columnar defects can be recast via the transfer matrix method in terms of the non-Hermitian quantum mechanics of bosons with a constant imaginary vector potential. ${ }^{3,4}$ Imaginary time replaces the $z$ direction chosen along the defect, and the bosons describe the vortices at each imaginary-time slice. A uniform imaginary vector potential creates a bias in the strength of the tunneling to one direction. For instance, a typical bosonic hopping term becomes $\mathbf{b}_{1}^{\dagger} \mathbf{b}_{2} e^{-h}+\mathbf{b}_{2}^{\dagger} \mathbf{b}_{1} e^{h}$, where $\mathbf{b}^{\dagger}, \mathbf{b}$ are the boson's creation and annihilation operators, and $i h$ is the imaginary vector potential. When $h>0$, hopping from site 1 to site 2 is suppressed, whereas hopping from 2 to 1 is enhanced. In a spatially periodic system, this may induce a transverse cur- rent of the bosons, which corresponds to wrapping of the vortices around the superconductor's periodic direction. When this happens, the energy eigenvalue of the nonHermitian Hamiltonian has an imaginary part, $\operatorname{Im} E$. The size of the transverse flux is proportional to the derivative $d \operatorname{Im} E / d h$ (see Sec. I A).

In the case of a thin superconducting slab, the mapping to non-Hermitian quantum mechanics leads to a onedimensional problem. In this case there has been recent progress on the breakdown of the transverse Meissner effect in the following specific problems: (1) Vortex hopping in a regular array of columnar pins with on-site and nearestneighbor repulsion. ${ }^{5,6}$ (2) Transmission through a weak link. ${ }^{5-7}$ (3) Vortex tunneling with Cauchy-distributed random

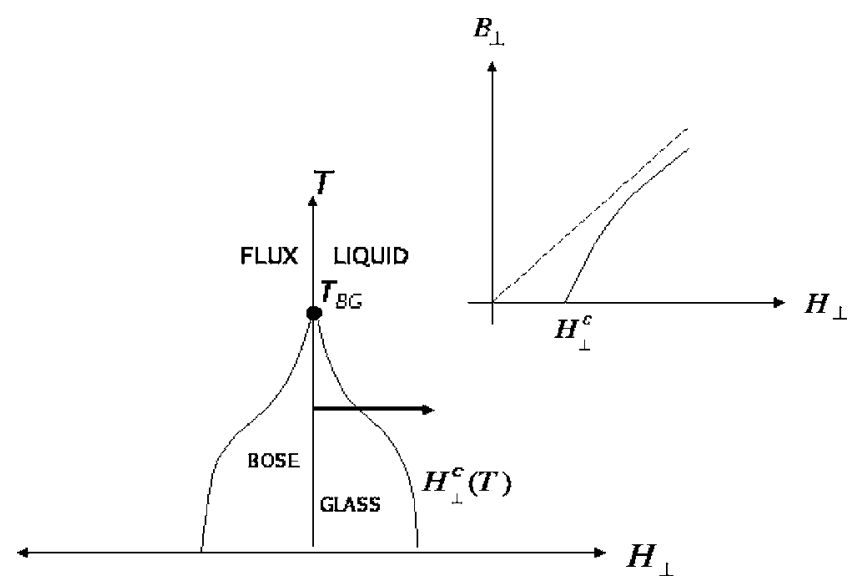

FIG. 1. Schematic phase diagram of a flux liquid with random columnar pins at a fixed external magnetic field $\vec{H}_{\|}$parallel to the pins as a function of temperature $T$ and tilted external field $H_{\perp}$. The Bose glass phase exhibits localized vortices and a transverse Meissner effect. The transitions along the line $H_{\perp}^{c}(T)$ map onto the nonHermitian quantum phase transition problem discussed in this paper. The inset shows the behavior of $B_{\perp}$ near $H_{\perp}^{c}$, where the transverse Meissner effect breaks down and the vortices begin to tilt. 
on-site pinning potential (Lloyd model). ${ }^{8-10}$ (4) Some progress has been made in understanding vortex dynamics with random on-site and random hopping energies in Refs. $11-13$.

In this paper we first concentrate on a fifth case, namely, random hopping in an otherwise uniform pinning array. The approach we take here is the strong randomness real-space renormalization group (RSRG). This approach, pioneered by $\mathrm{Ma}$, Dasgupta, and $\mathrm{Hu},{ }^{14,15}$ and developed further by Fisher, ${ }^{16,17}$ is very successful in treating one-dimensional random spin chains as well as other models. ${ }^{18-20}$ The use of this technique in the context of non-Hermitian transfer matrices, however, appears to be different.

This problem is of interest for several reasons. First, although much is known about non-Hermitian tight binding models with site randomness in $(1+1)$ dimensions, ${ }^{4,9-13}$ the case of pure bond randomness (corresponding to irregularly spaced columnar pins of equal strength in a slab) is relatively unexplored. All states are localized for the Hermitian problem of vortices in a thin slab without an external tilt field when only site randomness is present. The tilt field $h$ must then exceed a finite threshold before delocalized states appear in the center of the band. ${ }^{4}$ In contrast, there is always one delocalized state exactly at the band center for the Hermitian problem with only random hopping, accompanied by a diverging localization length of the localized states on either side. ${ }^{21}$

As we show explicitly in Sec. VII, additional delocalized states then appear immediately in the center of the band for any nonzero tilt in the thermodynamic limit. As discussed further below, for larger values of $h$ both problems do have similar mobility edges, separating localized states near the band edges from delocalized states near the band center.

Perhaps more important, the analytically tractable free fermion model discussed here sheds light on the poorly understood non-Hermitian quantum phase transition that describes the physics of vortices as one attempts to tilt them away from the direction preferred by columnar defects. Suppose for simplicity the longitudinal applied magnetic field (i.e., $H_{\|}$, the field parallel to the columnar pins) produces a vortex density which does not exceed the density of column pinning sites. Then, as shown in Fig. 1, the low-temperature Bose glass phase, with essentially all flux lines localized on columnar defects, is expected to be stable for a small additional field $H_{\perp}$ perpendicular to the column direction. The transverse applied field $H_{\perp}$ is proportional to the tilt field $h$ discussed in this paper. Below the zero tilt Bose glass transition temperature $T_{\mathrm{BG}}$, perpendicular fields less than a critical value $H_{\perp}^{c}$ leave vortices untilted and trapped on columnar pins in the thermodynamic limit. ${ }^{3}$ The equivalent quantum problem involves interacting quantum bosons in a disorder potential and constant imaginary vector potential proportional to $H_{\perp} \cdot{ }^{4}$ Provided the transition is not first order, the breakdown of this transverse Meissner effect above $H_{\perp}^{c}$ can be described by a critical exponent $\zeta$, according to

$$
B_{\perp} \propto\left(H_{\perp}-H_{\perp}^{c}\right)^{\zeta}
$$

where $B_{\perp}$ is the transverse flux due to the tilted vortices. Heuristic random walk arguments based on the entropy of

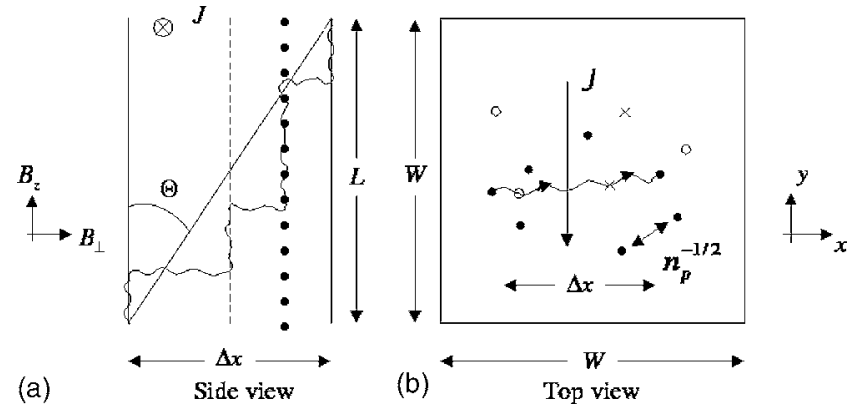

FIG. 2. (a) Schematic of a single tilted vortex line in a sample of thickness $L$ and inclined at an average angle $\theta$, interacting with a row of variable strength columnar pins (represented by solid dashed and dotted vertical lines) used in our estimate of the flux flow resistivity in the presence of a current $J$ flowing into the plane of the diagram. (b) Top view of this same situation, showing the path of the tilted vortex projected down the $z$ axis. $n_{p}$ is the density of columnar pins.

vortices wandering in the presence of thermal fluctuations lead to the estimates ${ }^{22}$

$$
\begin{aligned}
& \zeta=\frac{3}{2} \quad(d=3), \\
& \zeta=\frac{1}{2} \quad(d=2)
\end{aligned}
$$

in three and two dimensions, respectively. However, there are reasons to doubt these predictions. First, as will become clear, at least for the exactly soluble $(1+1)$-dimensional model discussed in this paper, the non-Hermitian quantum phase transition induced by tilt effectively occurs at a finite wave vector, calling into question simple random walk arguments based on physics at $k=0$. Second, although the phase diagram shown in Fig. 1 is well established experimentally, ${ }^{23-25}$ the one existing experimental measure of the exponent $\zeta$ (Ref. 26) (based on current-voltage characteristics, see below) is inconsistent with Eq. (2). Indeed, the experiments on bulk superconductors with columnar pins in Ref. 26 find $\zeta=1 / 2$, which disagrees with the prediction of Eq. (2) in $d=3$. In fact, we show in this paper that, for the special case of free fermions in $(1+1)$ dimensions (corresponding to Luttinger liquid parameter $g=1$ in Fig. 4) one obtains

$$
\zeta=1 \text { (free fermions, } 1+1 \text { dimensions), }
$$

which disagrees with the prediction of Eq. (2) in $d=2$.

In principle it might be possible to check predictions such as those in Eqs. (2) and (3) by magnetic torque measurements, which are sensitive to the differences in the direction of $\vec{B}$ and $\vec{H}$ that are an essential part of the transverse Meissner effect. ${ }^{24}$ Alternatively, as discussed in Refs. 3 and 22, the exponent $\zeta$ determines a density of kink excitations connecting nearby columnar defects (see Fig. 2), which in turn control the linear flux flow resistivity above $H_{\perp}^{c}$. Current-voltage curves are highly nonlinear below $H_{\perp}^{c}$, and the linear resistivity vanishes. ${ }^{3}$ To understand the linear resistivity above 
$H_{\perp}^{c}$, consider the geometry shown in Fig. 2(a), where a current flows perpendicular to the plane defined by the column direction and the average field direction defined by a set of tilted vortex lines. Imagine first a bulk sample, of dimensions $L \times W \times W$, where $L$ is the sample length along the columns. Suppose this field is inclined at a small angle $\theta=B_{\perp} / B_{\|}$away from the column direction. Then a typical perpendicular distance $\Delta x$ traversed by a vortex across a sample is $\Delta x=\theta L$. If the density of columnar pins is $n_{p}$, this tilt leads to

$$
N_{1}=\Delta x / n_{p}^{-1 / 2}=\frac{B_{\perp}}{B_{\|}} n_{p}^{1 / 2} L
$$

kinks associated with a single vortex. In clean type-II superconductors, at temperatures high enough so that residual pinning by point impurities can be neglected, these kinks will slide along columns, due to the Lorentz force $f_{L}=\phi_{0} J / c$ caused by the current, where $\phi_{0}$ is the flux quantum and $c$ the speed of light. Upon multiplying by the total number of flux lines $W^{2} B_{\|} / \phi_{0}$, we find a gas of kinks with density

$$
n_{k}=B_{\perp} n_{p}^{1 / 2} / \phi_{0}
$$

per unit volume. As expected, $n_{k}$ is proportional to $B_{\perp}$, which leads using Eq. (1) to a flux flow resistivity

$$
\rho=\rho_{0} B_{\perp} \xi^{2} / \phi_{0} \propto\left(H_{\perp}-H_{\perp}^{c}\right)^{\zeta},
$$

where $\rho_{0}$ is the normal state resistivity, $\xi$ (the coherence length) is the size of the normal vortex cores which contribute to the dissipation, and we have neglected coefficients of order unity. A similar calculation can be carried out for a two-dimensional slab with dimensions $L \times W \times d$, with $d$ $\ll L, W$ and a single sheet of columnar pins along $L$ with spacing $n_{p}^{-1}$. We also require $d<\lambda$, where $\lambda$ is the London penetration depth. We then find that the flux flow resistivity for a current perpendicular to the slab (i.e., along the direction $d$ ) is identical in form to the three-dimensional result (6). We hope that the calculations in this paper, although only valid in $(1+1)$ dimensions at a special temperature deep within the Bose glass phase (see Fig. 4), will stimulate further theory as well as experiments aimed at a more complete determination the exponent $\zeta$, in both two and three dimensions.

\section{A. Statement of the problem}

When a magnetic field is applied to a planar superconductor with parallel columnar defects in the plane, a competition occurs between the tendency of the vortices to be pinned by the columnar defects, and the portion of the inplane magnetic field which is normal to the columnar defects. The resulting lock-in of vortex trajectories parallel to the columns up to a critical tilt field is the transverse Meissner effect discussed above.

In this work we concentrate on a model of vortex hopping in a random array of columnar defects (see Fig. 3). As described previously, ${ }^{3,4}$ this statistical mechanics problem is mapped onto a quantum mechanical boson-hopping problem. When the magnetic field that produces the vortices is tilted relative to the columns, our model introduces non-

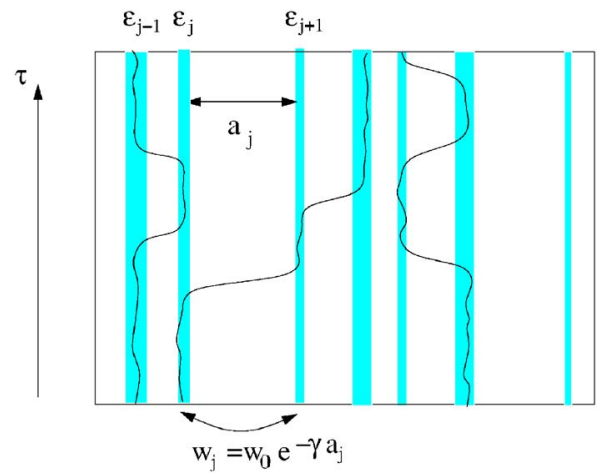

FIG. 3. (Color online) The exponential of the Hamiltonian (7) determines the transfer matrix describing the statistical mechanics of vortices (black lines) fluctuating in a planar superconductor with parallel columnar defects piercing it at random intervals (gray stripes). The distance between the defects $a_{j}$, as well as their energy depth (illustrated by their width) $V_{j}$, are random variables. The irregular spacing leads to strong randomness in the vortex hopping $w_{j} \propto e^{-\gamma a_{j}}$. The external magnetic induction and its direction relative to the columnar pins determine the vortex chemical potential $\mu$ and the effective tilt $h_{j} \propto a_{j}$. In this paper we compare and contrast the case of identical pins with random spacings, and the case of random-pinning energies but uniform spacings.

Hermiticity to the Hamiltonian. The Hamiltonian whose exponential gives the transfer matrix is

$$
\begin{aligned}
\mathcal{H}= & \sum_{i}\left[-w_{i}\left(\mathbf{b}_{i}^{\dagger} \mathbf{b}_{i+1} e^{-h_{i}}+\mathbf{b}_{i+1}^{\dagger} \mathbf{b}_{i} e^{h_{i}}\right)\right. \\
& \left.+\left(\epsilon_{i}-\mu\right) \mathbf{b}_{i}^{\dagger} \mathbf{b}_{i}+\frac{U}{2} n_{i}\left(n_{i}-1\right)\right] .
\end{aligned}
$$

Each vortex is represented by a boson with creation and annihilation operators $\mathbf{b}, \mathbf{b}^{\dagger}$. As discussed above, we restrict our attention to hard-core vortices, and set the on-site repulsion to infinity $(U \rightarrow \infty)$.

The average number $n$ of vortices per pinning site is equivalent to the longitudinal magnetization induced by a magnetic field $H_{\|}$parallel to the defects. The part of the magnetic field $H_{\perp}$, tilted relative to the columnar defects, induces an imaginary vector potential $h_{i}$ on each bond. We expect it to depend linearly on the distance between columnar pins. Note, however, that using a similarity transformation one can redistribute the $h_{i}$ 's such that each bond carries the same imaginary vector potential. ${ }^{11,12}$ In the following we will therefore eventually apply a uniform tilt $h$ for all bonds. In Eq. (7), $\mu$ is the vortex chemical potential, which is controlled by the external magnetic field and the depth of the pinning potential. The hopping energies $w_{i}$ and pinning energies $\epsilon_{i}$ are random variables with some relatively well behaved distribution. As is shown schematically in Fig. 3, the Hamiltonian (7) describes the statistical mechanics of vortices hopping between columnar defects with an external magnetic induction tilted relative to the columnar pins.

In general, our goal is to characterize the transverse Meissner effect in the superconductor. For that purpose we also need to define the transverse magnetic flux in terms of 


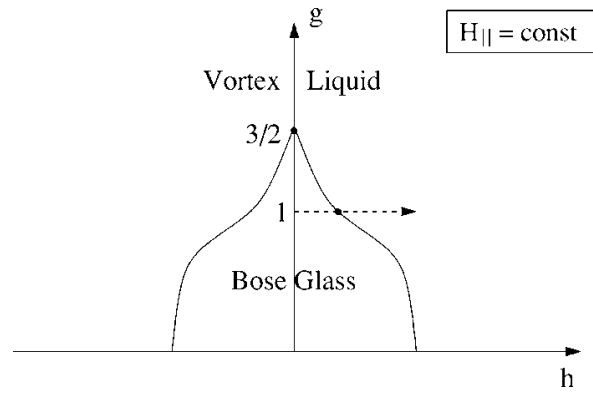

FIG. 4. The phase diagram for interacting vortices, with effective Luttinger parameter $g$ proportional to temperature [see Eq. (9)], tilt $h$, at a fixed parallel magnetic field $H_{\|}$. For real bosons, the vortex liquid phase for $g>3 / 2$ and vanishing tilt $h=0$ corresponds to a superfluid with off-diagonal long range order. Little is known about the Bose glass depinning transition at finite $h$. In this paper we investigate this transition at $g=1$.

the bosons of Eq. (7). Quite intuitively, the transverse flux is equivalent to the boson current, and is found by differentiating the Hamiltonian with respect to the external transverse field $^{3,4}$

$$
\mathbf{J}_{i}=(-i) \frac{\partial \mathcal{H}}{\partial h_{i}}=(-i) w_{i}\left(\mathbf{b}_{i}^{\dagger} \mathbf{b}_{i+1} e^{-h_{i}}-\mathbf{b}_{i+1}^{\dagger} \mathbf{b}_{i} e^{h_{i}}\right) .
$$

We will consider and contrast two cases: first, the case of random-pinning energies with uniform spacings, and then the case of identical pins but random spacings. We will show that these two cases are quite different, and proceed to characterize the case of random spacings in some detail.

As we show in Sec. II B, the Hamiltonian (7) with the hard-core requirement $(U \rightarrow \infty)$ is equivalent to a fermion model without interactions. In general these interactions are quantified by the Luttinger parameter ${ }^{6}$

$$
g \sim \frac{\pi T}{\sqrt{C_{11} C_{44}}},
$$

where $T$ is the temperature and $C_{11}$ and $C_{44}$ are the vortex compressibility and tilt modulus, respectively. In this work we concentrate on the line $g=1$, and ignore nearest neighbor and higher range interactions between the hard-core vortices. The general phase diagram for the interacting vortex lines with tilt in $(1+1)$ dimensions is shown in Fig. 4..$^{3,22}$

\section{B. Summary of results}

In the first part of this paper (Secs. II and III) we review and derive properties of the random-pinning Lloyd model. We concentrate on the minimum tilt required to form delocalized eigenstates, i.e., the critical tilt as a function of parallel field to destroy the transverse Meissner effect, and the resulting transverse flux as a function of both tilt and parallel field. The derivation of these properties provides us with a baseline for a comparison of the random-pinning model with the random-hopping model.

In the second part of the paper we concentrate on random hopping. In this model all pinning sites are assumed to be identical, but with random distances between them (since hopping depends exponentially on the intersite distance, the hopping strengths $w_{i}$ will be strongly random). For this purpose we employ the real-space renormalization group (RSRG) method. Using this method we obtain the density of states for the vortex-hopping Hamiltonian. We derive this method for the vortex-hopping problem in Sec. IV.

The random-hopping problem has two unique features that are connected to each other: the localization length of the vortex eigenfunctions diverges near the middle of the band $(E=0)$, and there is also a singularity of the DOS at the same place. We use the DOS to derive a relationship between the effective vortex chemical potential $\mu$ and the applied parallel field $B_{\|}$. We then proceed to show that any nonzero tilt will produce delocalized eigenstates, and derive the critical tilt $h_{c}(b)$ at which the transverse Meissner effect breaks down. We also derive the localization length of vortex states near the mobility edge.

By employing a simple spectral formula explored in Refs. 9,11 , and 12 , together with the results of the real-space $R G$ and the general arguments in Appendix A, we derive the following properties: the angle of approach of the delocalized spectrum in the complex plane $(d \operatorname{Im} E / d \operatorname{Re} E)$, the contribution to the transverse magnetization of a single delocalized vortex state, and the total vortex current, or transverse flux, near the breakdown of the transverse Meissner effect.

We emphasize that the results derived here assume that the system is in the universal low-energy limit. A numerical investigation of this limit requires large system sizes that allow the RSRG to reach low energies. This is confirmed in Sec. VII where finite-size systems of vortices and columnar pins with random pinning and random hopping are diagonalized exactly.

\section{EQUIVALENT MODELS}

Hamiltonian (7) in the limit $U \rightarrow \infty$ is not as familiar to us as some other equivalent models. In this section we map the boson Hamiltonian (7) to a spin model with easy plane anisotropic interactions $(X X)$, and to a fermion-hopping model. These mappings will be useful when applying the RSRG in Sec. IV and exact diagonalization for finite systems in Sec. VII.

\section{A. Mapping to a spin model}

By the simple transformation of the boson operators on the $n$th lattice site

$$
\begin{gathered}
\mathbf{b}_{n}^{\dagger}=(-1)^{n} \cdot \hat{S}_{n}^{+}=(-1)^{n} \cdot\left(\hat{S}_{n}^{x}+i \hat{S}_{n}^{y}\right), \\
\mathbf{b}_{n}=(-1)^{n} \cdot \hat{S}_{n}^{-}=(-1)^{n} \cdot\left(\hat{S}_{n}^{x}-i \hat{S}_{n}^{y}\right), \\
\mathbf{b}_{n}^{\dagger} \mathbf{b}_{n}=1 / 2+\hat{S}_{n}^{z},
\end{gathered}
$$

the Hamiltonian in Eq. (7) is transformed to an $X X$ ferromagnet in an external magnetic field 


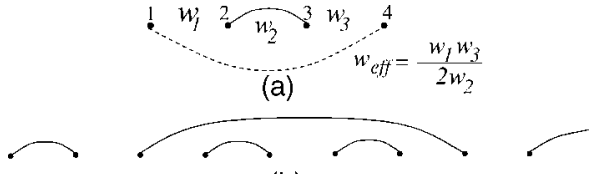

(b)

FIG. 5. (a) In a random $X X$ spin chain, described by Eq. (11) with $h=0$, strong bonds such as $w_{2}$ localize a spin singlet. Quantum fluctuations induce a coupling between the neighbors of the singlet. This coupling has the same $X X$ form, but a substantially reduced scale $w_{\text {eff }}=w_{1} w_{3} / w_{2}$ which is much smaller than $w_{1}, w_{2}$, and $w_{3}$. By repeating the singlet formation process, the energy scale of the effective Hamiltonian is reduced. (b) The random singlet state. Singlets form in a random fashion, mostly between nearest neighbors, but they also connect very far away spins. Long distance singlets give rise to average correlations that decay algebraically with distance.

$$
\begin{aligned}
\mathcal{H}= & \sum_{i}\left\{-2 w_{i}\left[\left(\hat{S}_{i}^{x} \hat{S}_{i+1}^{x}+\hat{S}_{i}^{y} \hat{S}_{i+1}^{y}\right) \cosh (h)\right.\right. \\
& \left.\left.+i\left(\hat{S}_{i}^{x} \hat{S}_{i+1}^{y}-\hat{S}_{i}^{y} \hat{S}_{i+1}^{x}\right) \sinh (h)\right]+\left(\epsilon_{i}-\mu\right)\left(\frac{1}{2}+\hat{S}_{i}^{z}\right)\right\} .
\end{aligned}
$$

In the spin variables, a vortex pinned at site $i$ corresponds to $\hat{S}_{i}^{z}=+1 / 2$; an empty site $i$ is transformed to a site with $\hat{S}_{i}^{z}=$ $-1 / 2$. The transformation in Eq. (11) is the special spin-1/2 case of the Holstein-Primakoff transformation. ${ }^{27}$ In its higher spin version it has factors of the form $\sqrt{1-\mathbf{b}_{n}^{\dagger} \mathbf{b}_{n}}$ which for spin-1/2 reduce to either zero or one.

At half filling (i.e., when $\mu=\epsilon_{i}=0$ ) and zero tilt $h$ the ground state of Hamiltonian (11) is the random singlet phase. In this phase singlets form in a random fashion between sites connected by strong $w_{i}$. Once a singlet forms, the nearest neighbors of the two sites involved also interact, but with suppressed strength. By pairing up strongly interacting sites into singlets, we iteratively reduce the energy scale of the Hamiltonian, until we exhaust all sites. At this point, singlets connect many nearest neighbors, but occasionally singlets connect very far away spins, leading to power-law decaying correlations (for a review, see Ref. 16). The idea behind the random singlet phase and its formation are shown in Fig. 5. A thorough discussion of this phase and its implications for vortex pinning will be given in Secs. IV and V.

\section{B. Mapping to fermion-hopping problem}

By using a version of the Wigner-Jordan transformation, ${ }^{28}$ we can also map the hard-core boson Hamiltonian (7) with $U=\infty$, to a fermionic random-hopping problem. This mapping is also quite straightforward; we start with

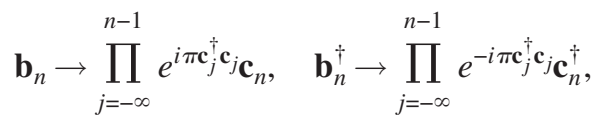

where the string operator ensures the anticommutation relations of the $c_{n}$ 's. Now we can write the Hamiltonian of the vortices as though they are fermions

$$
\mathcal{H}=\sum_{i}\left[-w_{i}\left(\mathbf{c}_{i}^{\dagger} \mathbf{c}_{i+1} e^{-h_{i}}+\mathbf{c}_{i+1}^{\dagger} \mathbf{c}_{i} e^{h_{i}}\right)+\left(\epsilon_{i}-\mu\right) \mathbf{c}_{i}^{\dagger} \mathbf{c}_{i}\right]
$$

Similarly, the local current operator becomes

$$
\begin{aligned}
\mathbf{J}_{i} & =(-i) \frac{\partial \mathcal{H}}{\partial h_{i}}=(-i) w_{i}\left(\mathbf{b}_{i}^{\dagger} \mathbf{b}_{i+1} e^{-h_{i}}-\mathbf{b}_{i+1}^{\dagger} \mathbf{b}_{i} e^{h_{i}}\right) \\
& =(-i) w_{i}\left(\mathbf{c}_{i}^{\dagger} \mathbf{c}_{i+1} e^{-h_{i}}-\mathbf{c}_{i+1}^{\dagger} \mathbf{c}_{i} e^{h_{i}}\right) .
\end{aligned}
$$

In the absence of the tilt field, the ground state of the Hamiltonian (13) is well understood. ${ }^{16}$ When the pins are identical $\left(\epsilon_{i}=0\right)$, the random hopping localizes all states $e x$ cept at half filling, where there is always a delocalized state. The localized states away from the mobility edge are related to the random singlets in the $X X$ spin chain of Eq. (11). Instead of a singlet, however, pairs of sites share a single fermion.

In this model, half filling is obtained when $\mu \rightarrow 0^{-}$. In this limit, the last fermion inserted in the system is delocalized between two sites with a distance that is of the order of the system size, i.e., it is delocalized. All other fermionic states, however, are localized.

In the presence of the tilt field $h$ an entire band of delocalized states appears, and the lower mobility edge moves down to fillings below one half, and to negative chemical potentials $\mu<0$. By using the mapping to free fermions [Eq. (13)] and the known real space renormalization group (RSRG) results for this model, we can obtain much insight into the delocalized phase. In particular, we will derive a universal relationship between the field $h$, the mobility edge $\mu_{h}$, and perhaps most importantly, the vortex density (i.e., parallel magnetic field) at the mobility edge $\rho_{h}$.

\section{RANDOM-PINNING ENERGY AND UNIFORM HOPPING-EXACT RESULTS FROM THE LLOYD MODEL}

In this section we will review exact results for the spectrum of the non-Hermitian Lloyd model, ${ }^{8-10}$ and proceed to derive results in the presence of a transverse magnetic field (Sec. III B). These results present another reference point for the behavior of the transverse magnetization near the breakdown of the transverse Meissner effect, and go beyond the Lloyd model.

We consider the one-dimensional quantum Hamiltonian (7) with infinite on-site repulsion $(U \rightarrow \infty)$ :

$$
\mathcal{H}=\sum_{i}\left[-w\left(\mathbf{b}_{i}^{\dagger} \mathbf{b}_{i+1} e^{-h}+\mathbf{b}_{i+1}^{\dagger} \mathbf{b}_{i} e^{h}\right)-\left(\boldsymbol{\epsilon}_{i}-\mu\right) \mathbf{b}_{i}^{\dagger} \mathbf{b}_{i}\right],
$$

where $\mathbf{b}_{i}^{\dagger}$ and $\mathbf{b}_{i}$ are creation and annihilation operators of hard-core bosons, which represent the vortices. The hopping matrix element $w$ is now site independent. We could equally well use the fermion representation in Eq. (13) in what follows. We set the lattice constant to 1 , and consider a lattice of length $L$ sites. For small tilts, the imaginary gauge field $h$ is proportional to the angle of the applied magnetic field relative to the columnar defects. The parallel applied field translates into the chemical potential for the vortices via the constraint 


$$
\int_{-\infty}^{\mu} g(\epsilon) d \epsilon=n
$$

where $n$ is the average number of vortices per columnar defect and $g(\epsilon)$ is the density of states associated with the Hamiltonian of Eq. (15). Assuming that the distribution of pinning energies $\epsilon_{i}$ is symmetric, we can invoke particle-hole symmetry, and rewrite Eq. (16) as

$$
\int_{\mu}^{0} g(\epsilon) d \epsilon=\int_{-\infty}^{0} g(\epsilon) d \epsilon-\int_{-\infty}^{\mu} g(\epsilon) d \epsilon=0.5-n .
$$

In this section we will review the known qualitative feature of this noninteracting model of vortices with random pinning strength $\epsilon_{i}$. We will also focus on the analytic results known for the Lloyd model, ${ }^{8-10}$ which is described by the Hamiltonian (15) with a special distribution of the pinning energies $\epsilon_{i}$

$$
P[\epsilon]=\frac{\gamma}{\pi} \frac{1}{\epsilon^{2}+\gamma^{2}} .
$$

This model has played an important role in our understanding of one-dimensional localization of electrons.

\section{A. Structure of the spectrum and critical delocalization tilt}

Much of the physics of interacting vortices in $(1+1)$ dimensions can be inferred from the shape of the spectrum of the Hamiltonian as a function of the applied transverse field $h$. For zero tilt $h$, the entire spectrum of Hamiltonian (15) is due to localized states, and is therefore real. ${ }^{4}$ The density of states changes as a function of energy but experiences no singularities. When the external magnetic field is tilted relative to the columnar defects, the spectrum of the localized states does not change, but the wave functions associated with them spread out in the direction of the tilt. The invariance of the spectrum to the tilt, as long as the wave functions are localized, can be easily understood, since for any eigenstate of the Hamiltonian we can carry out a gauge transformation such that all the imaginary gauge field in a sufficiently large system is on a bond between sites where the eigenstate has no support.

Above some critical tilt $h_{0}>0$, a subset of the eigenfunctions delocalizes, and their energies become complex (see Fig. 6). As shown in Refs. 11 and 12, the parts of the spectrum that first become delocalized are at the maximum of the DOS $g(\epsilon)$, which is generally in the center of the spectrum, at $\epsilon=0$.

The transverse Meissner effect, however, persists until the vortex-eigenstates at the "Fermi energy" $\mu$ become delocalized. This Fermi energy for the vortices is determined by the parallel (longitudinal) magnetic field $B_{\|}$or, equivalently, by the vortex density $n$ per pin. The tilt at which the transverse Meissner effect breaks down is thus a function of $n$, and it is always bigger than the threshold tilt $h_{0}$ :

$$
h_{c}(n) \geqslant h_{0} \text {. }
$$

The qualitative description above is exemplified by the spectrum of the Lloyd model. ${ }^{8,9}$ The delocalized portion of (a)

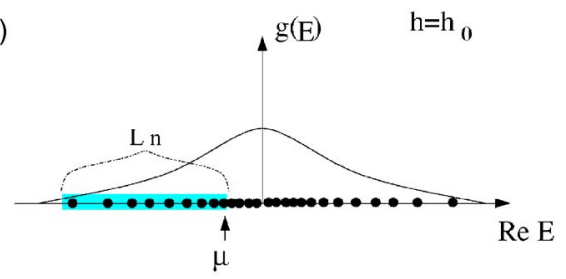

(b)

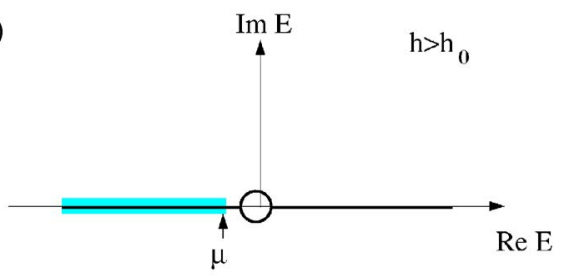

(c)

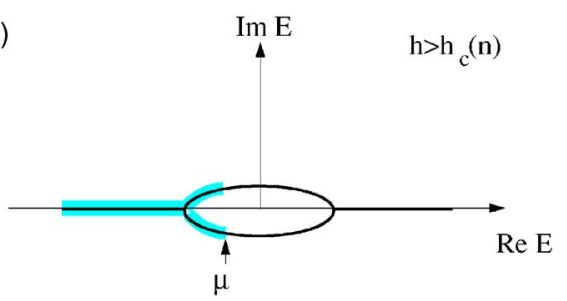

FIG. 6. (Color online) (a) All eigenfunctions of the randompinning Hamiltonian are localized and independent of the tilt provided $h<h_{0}$. We suppose that the density of states (DOS) is peaked around $E=0$ but is not singular. The effective chemical potential of the vortices, $\mu$, is determined by the longitudinal magnetization $n$, such that the density of occupied states below $\mu$ equals $n$. (b) When the tilt of the applied field becomes $h>h_{0}$, a bubble of delocalized states develops where the DOS is peaked. As long as the bubble does not reach $\mu$, there is still no transverse magnetization. The thick lines mark the support of the eigenenergies in the complex $E$ plane. (c) The transverse Meissner effect breaks down when $h=h_{c}$, and the delocalized bubble reaches $\mu$. The total vortex current is shown in Appendix A to be proportional to the imaginary part of the energy eigenvalue at the Fermi surface [see Eq. (A4)].

the spectrum of the Lloyd model is known to $\mathrm{be}^{8}$

$$
E_{n}^{ \pm}=-2 w \cos \left( \pm k_{n}+i h\right) \mp i \gamma,
$$

where $\gamma$ controls the width of the site-randomness distribution in Eq. (18). As the tilt increases such that $h>h_{0}$, a bubble of delocalized states appears in the center of the band (see Fig. 6). When $h>h_{c}(n)$, this bubble engulfs the chemical potential.

From the spectrum of the Lloyd model (20), one can easily derive $h_{0}$ and $h_{c}$. The lowest critical tilt $h_{0}$ that produces delocalized eigenfunctions is obtained when the imaginary part of the energy becomes nonzero, $\operatorname{Im} E_{n} \neq 0$, for some eigenstate $E_{n}$. As is obvious from Fig. 6(b), this delocalization tilt will be the critical tilt where the transverse Meissner effect disappears at half filling $(n=0.5) . h_{0}$ is given by

$$
\sinh \left(h_{0}\right)=\frac{\gamma}{2 w} .
$$

The range of wave vectors $k_{n}$ that defines the delocalized states are thus given by 


$$
\sin \left|k_{n}\right|>\frac{\gamma}{2 w \sinh (h)}
$$

To obtain the critical tilt as a function of longitudinal magnetic field, we observe that the number of delocalized singlevortex states is

$$
N_{d e l}=\frac{2 L}{\pi} \arccos \left(\frac{\gamma}{2 w \sinh (h)}\right)
$$

(note that the argument of the arccos is never bigger than 1). Thus the critical longitudinal vortex density for a given tilt is

$$
n_{c}=\frac{1}{2}-\frac{1}{\pi} \arccos \left(\frac{\gamma}{2 w \sinh (h)}\right),
$$

which can be inverted to give the critical tilt for a given magnetization

$$
\sinh \left(h_{c}\right)=\frac{\gamma}{2 w \cos [\pi(0.5-n)]}
$$

\section{B. Transverse magnetic flux near critical tilt}

Once vortices form delocalized states, the magnetization in the superconductor is no longer parallel to the columnar defects, and transverse flux appears. The total transverse flux, or vortex current in the quantum-mechanical picture, can also be found out easily for the Lloyd model. ${ }^{8}$

In Appendix A we derive a general rule for the total vortex current for delocalized states [Eq. (A4)]:

$$
J_{\text {total }}=2 \frac{1}{L} \sum_{k_{n}} \operatorname{Re} \frac{\partial E_{n}}{\partial h}=\frac{1}{\pi}\left(\operatorname{Im} E_{\mu}-\operatorname{Im} E_{\mu_{m}}\right),
$$

where $\operatorname{Im} E_{\mu}$ is the imaginary part of the energy eigenvalue with real part $\mu$ and $\mu_{m}(h)$ is the chemical potential above which vortex states are delocalized (mobility edge). $L$ is the total number of lattice sites. As discussed in Refs. 4 and 6, this total "current" (similar to the derivative with respect to vector potential which gives the current in a quantum system) is proportional to the perpendicular component of the magnetic flux. If we consider our system having longitudinal vortex density $n$ and tilt $h>h_{c}$, then the number of delocalized vortices (as opposed to vortex states) is $n-n_{c}$ where $n_{c}$ is given in Eq. (24). Therefore, the $k_{n}$ that are occupied by delocalized vortices are given by

$$
\begin{aligned}
& \arcsin \left(\frac{\gamma}{2 w \sinh (h)}\right) \\
& \quad=k_{c}<\left|k_{n}\right|<\arcsin \left(\frac{\gamma}{2 w \sinh (h)}\right)+\pi\left(n-n_{c}\right)=k_{F} .
\end{aligned}
$$

Now, using Eq. (A4) (see Appendix A) we can directly write the total current as

$$
\begin{aligned}
J_{\text {total }}= & \frac{1}{\pi}\left(\operatorname{Im} E_{k_{F}}-\operatorname{Im} E_{k_{c}}\right) \\
= & \frac{1}{\pi} 2 w \sinh (h)\left\{\sin \left[\arcsin \frac{\gamma}{2 w \sinh (h)}+\pi\left(n-n_{c}\right)\right]\right. \\
& \left.\quad-\sin \left(\arcsin \frac{\gamma}{2 w \sinh (h)}\right)\right\} \\
= & \frac{1}{\pi} 2 w \sinh (h)\left(\frac{\gamma}{2 w \sinh (h)}\left\{\cos \left[\pi\left(n-n_{c}\right)\right]-1\right\}\right. \\
& \left.+\sqrt{1-\frac{\gamma^{2}}{(2 \sinh (h) w)^{2}}} \sin \left[\pi\left(n-n_{c}\right)\right]\right)
\end{aligned}
$$

Near the delocalization transition we have

$$
J_{\text {total }}=2 w \sinh (h) \sqrt{1-\left(\frac{\gamma}{2 w \sinh (h)}\right)^{2}}\left(n-n_{c}\right) .
$$

Using the derivative of Eq. (24) we obtain

$$
\frac{d n_{c}}{d h}=\frac{1}{2 \pi} \frac{\gamma}{2 w} \frac{\cosh (h)}{\sinh ^{2} h} \frac{1}{\sqrt{1-\left(\frac{\gamma}{2 w \sinh (h)}\right)^{2}}} .
$$

Upon putting this back in Eq. (29) with $h-h_{c} \approx \mid d n / d h(n$ $\left.-n_{c}\right)$ | we obtain a simple expression for the total current in the Lloyd model near delocalization, namely,

$$
J_{\text {total }} \approx \frac{\gamma}{2 \pi \tanh (h)}\left(h-h_{c}\right)
$$

This linear onset of the vortex current is qualitatively similar to numerical results for a box distribution of pinning energies, as presented in Sec. VII. When reintegrated as a formula for the transverse magnetic flux of a vortex system, (30) leads to the prediction $\zeta=1$ for the exponent discussed in the Introduction, Eq. (2).

\section{REAL SPACE RG OF THE BOSON-HOPPING PROBLEM}

One purpose of this paper is to understand the properties of vortices hopping in an array of identical pins with random locations, and contrast these properties with those of the random-pinning energy system with uniform hopping. The random-hopping problem is described by the Hamiltonian in Eq. (7) with $U \rightarrow \infty, \epsilon_{i}=0$, and $w_{i}$ random. In order to solve this model we apply the real-space renormalization group (RSRG) procedure. ${ }^{14-17}$ In this section we review the application of the RSRG to the random-hopping boson Hamiltonian.

The RSRG allows us to diagonalize the random Hamiltonian iteratively by eliminating the high energy degrees of freedom. The first step is finding the strongest bond in the chain and diagonalizing it while ignoring the rest of the Hamiltonian. Quantum fluctuations give rise to new interactions that bridge over the bond we diagonalize. If we are lucky, these renormalized interactions will be of the same form as the original Hamiltonian. Let us carry this out for the 
boson-hopping Hamiltonian (7). In effect, we diagonalize the Hamiltonian (7) iteratively by putting vortices into a hierarchy of localized states shared by pairs of sites.

\section{A. Diagonalization of the strongest bond}

The Hamiltonian we are interested in is given in Eq. (7), with $U \rightarrow \infty$ and $\epsilon_{i}=0$ :

$$
\mathcal{H}=\sum_{i}\left[-w_{i}\left(\mathbf{b}_{i}^{\dagger} \mathbf{b}_{i+1} e^{-h}+\mathbf{b}_{i+1}^{\dagger} \mathbf{b}_{i} e^{h}\right)-\mu \mathbf{b}_{i}^{\dagger} \mathbf{b}_{i}\right] .
$$

Suppose $w_{i}$ is the strongest hopping energy in the chain. We first diagonalize a single nearest neighbor bond

$$
\mathcal{H}_{i}=-w_{i}\left(\mathbf{b}_{i}^{\dagger} \mathbf{b}_{i+1} e^{-h}+\mathbf{b}_{i+1}^{\dagger} \mathbf{b}_{i} e^{h}\right)-\mu\left(\mathbf{b}_{i}^{\dagger} \mathbf{b}_{i}+\mathbf{b}_{i+1}^{\dagger} \mathbf{b}_{i+1}\right) .
$$

Because the vortices are hard core in this limit the only occupied states available in the Hilbert space are

$$
|i\rangle=\mathbf{b}_{i}^{\dagger}|0\rangle, \quad|i+1\rangle=\mathbf{b}_{i+1}^{\dagger}|0\rangle .
$$

Diagonalizing Eq. (31) in the subspace spanned by Eq. (32) means diagonalizing the matrix

$$
\left(\begin{array}{cc}
-\mu & -w_{i} e^{-h} \\
-w_{i} e^{h} & -\mu
\end{array}\right)
$$

Note that the eigenvalues of this Hamiltonian cannot depend on the tilt, in accordance with what we know on localized states - they carry no transverse magnetic field. ${ }^{4}$ The eigenvalues and right eigenvectors of Eq. (33) are

$$
\begin{gathered}
E_{ \pm}=-\mu \pm w_{i}, \\
| \pm\rangle=\frac{1}{\sqrt{2}}\left(e^{-h / 2}|i\rangle \mp e^{h / 2}|i+1\rangle\right) .
\end{gathered}
$$

Note that in this non-Hermitian quantum problem, the left eigenvectors are not the Hermitian conjugates of the right ones. In fact,

$$
\langle \pm|=\frac{1}{\sqrt{2}}\left(e^{h / 2}\langle i| \mp e^{-h / 2}\langle i+1|\right) .
$$

As long as $E_{-}<0$ and $E_{+}>0$ the ground state of $\mathcal{H}_{i}$ from Eq. (31) will contain a single vortex split between sites $i$ and $i+1$. The eigenstate of bond $i$ is therefore

$$
|-\rangle=\left(e^{-h / 2} \mathbf{b}_{i}^{\dagger}+e^{h / 2} \mathbf{b}_{i+1}^{\dagger}\right)|0\rangle .
$$

This state is described in Fig. 7(a).

The next step is to include the rest of the Hamiltonian, and in particular neighboring bonds corresponding to $\mathcal{H}_{i \pm 1}$. These terms will lead to quantum fluctuations above the ground state of $\mathcal{H}_{I}$. Thus it is necessary to find the allowed excited states of Eq. (7) which involve 2 and 0 vortices. These states are simply

$$
\begin{gathered}
|1,1\rangle=\mathbf{b}_{i}^{\dagger} \mathbf{b}_{i+1}^{\dagger}|0\rangle, \\
|0,0\rangle=|0\rangle,
\end{gathered}
$$

with energies $E=-2 \mu$ and $E=0$, respectively; they are depicted in Figs. 7(b) and 7(c). Note that their kinetic energy is

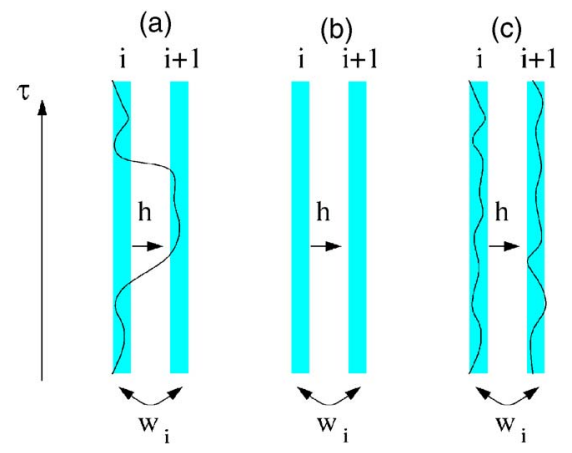

FIG. 7. (Color online) Eigenstates of the bond $i$. (a) The lowest eigenstate of the Hamiltonian $\mathcal{H}_{i}$ from Eq. (31) is $|-\rangle$ [Eq. (35)]. Sites $i$ and $i+1$ share a single vortex. In the imaginary time description, it is as though the vortex tunnels back and forth between the two columnar pins. The energy of this state is $-\mu-w_{i}$. (b) An excited state in which both pins are empty, with energy eigenvalue zero. (c) An excited eigenstate of $\mathcal{H}_{i}$ with energy $-2 \mu$; both pins are occupied by vortices.

$E_{k}=0$; their only energy comes from the chemical potential.

\section{B. Second order perturbation theory and renormalized hopping and tilt}

Having diagonalized the bond $\mathcal{H}_{i}$ we now need to incorporate the rest of the Hamiltonian as a perturbation. To second order in the hopping matrix elements, we need to concentrate only on the two neighboring bonds

$$
\begin{aligned}
V= & -w_{i-1}\left(\mathbf{b}_{i-1}^{\dagger} \mathbf{b}_{i} e^{-h}+\mathbf{b}_{i}^{\dagger} \mathbf{b}_{i-1} e^{h}\right) \\
& -w_{i+1}\left(\mathbf{b}_{i+1}^{\dagger} \mathbf{b}_{i+2} e^{-h}+\mathbf{b}_{i+2}^{\dagger} \mathbf{b}_{i+1} e^{h}\right) .
\end{aligned}
$$

The second order Hamiltonian is then

$$
\begin{aligned}
V^{(2)} & =-\frac{\langle-|V| 0,0\rangle\langle 0,0|V|-\rangle+\langle-|V| 1,1\rangle\langle 1,1|V|-\rangle}{w_{i}} \\
& =-\frac{w_{i-1}^{2}}{w_{i}}-\frac{w_{i+1}^{2}}{w_{i}}-\frac{w_{i-1} w_{i+1}}{w_{i}}\left(\mathbf{b}_{i-1}^{\dagger} \mathbf{b}_{i+2} e^{-3 h}+\mathbf{b}_{i+2}^{\dagger} \mathbf{b}_{i-1} e^{3 h}\right),
\end{aligned}
$$

where, as in Eq. (36), $|0,0\rangle$ is a state with both sites $i$ and $i$ +1 empty, and $|1,1\rangle$ is a state with both sites full. Quantum fluctuations thus give rise to a new coupling between sites $i-1$ and $i+2$. This renormalization process is depicted in Fig. 8, and is discussed below.

Equation (38) is quite remarkable. It tells us that after localizing a vortex between sites $i$ and $i+1$, we can forget about these two sites. Except for a constant contribution, the only change we need to make to the Hamiltonian is to add a new hopping term that allows vortices to hop over the occupied pair, from $i-1$ to $i+2$. In addition, we see that this hopping is associated with the composite tilt of the three bonds linking $i-1$ and $i+2$. In general, upon allowing for different tilt fields on neighboring bonds, the effective strength and effective tilt of the renormalized bond are, respectively, 


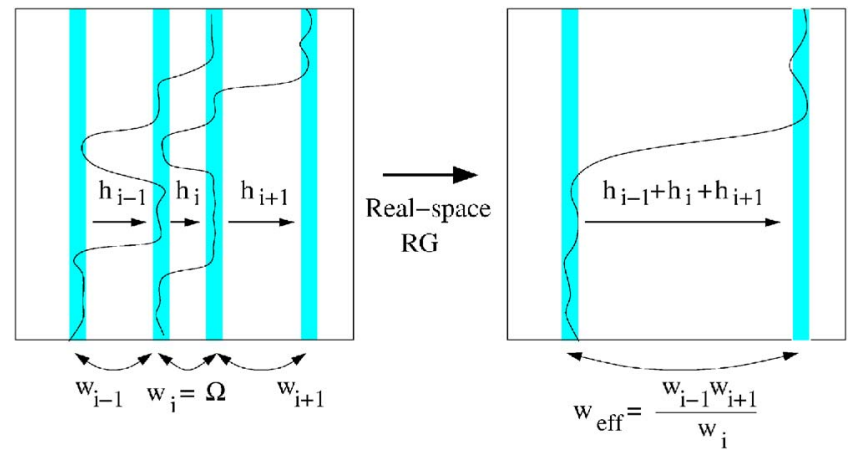

FIG. 8. (Color online) Real-space RG decimation step, where we allow for different tilt fields $h_{j}$ on neighboring bonds. In each step of the renormalization we find the strongest bond $w_{i}=\Omega$. We diagonalize it and minimize its energy by allowing a single vortex to fluctuate between the two columnar pins $i$ and $i+1$. A vortex in site $i-1$ may then fluctuate into site $i$, raise the energy of sites $i$ and $i+1$, and make the vortex in site $i+1$ relax by fluctuating into site $i+2$. Thus we can eliminate sites $i$ and $i+1$, by including the effective hopping between sites $i-1$ and $i+2$. The effective transverse field (tilt) between $i-1$ and $i+2$ becomes the sum of the tilts connecting these two sites.

$$
\begin{gathered}
w_{i-1, i+2}^{\mathrm{eff}}=\frac{w_{i-1} w_{i+1}}{\Omega}, \\
h_{i-1, i+2}^{\mathrm{eff}}=h_{i-1}+h_{i}+h_{i+1},
\end{gathered}
$$

where we introduced the notation $\Omega=\max \left\{w_{i}\right\}$ (also see Fig. 8). Equations (39) are the $R G$ rules for the RSRG as applied to the vortex-hopping problem.

As it turns out, Eqs. (39) coincide with the RG flow equations for the fermion-hopping problem (or $X X$ spin chain problem) in the Hermitian case $h=0$. The only difference is that the tilt $h_{i}$ replaces the length of bonds, as one might expect in the case of localized states. ${ }^{4}$ In order to solve the flow equations we can thus use techniques known from the Hermitian case.

Two important points should be made here. (1) The flows of the $h_{i}$ 's and $w_{i}$ 's are independent. (2) No new terms are produced. In particular, we do not produce randomness in the chemical potential. In the pure model it is the particle-hole symmetry that prevented us from having new chemicalpotential terms. But Eq. (7) with nonzero tilt does not have particle-hole symmetry or, more precisely, it is symmetric under a particle-hole transformation accompanied by a changing the tilt direction

$$
\mathbf{b} \rightarrow \mathbf{b}^{\dagger}, \quad \mathbf{b}^{\dagger} \rightarrow \mathbf{b}, \quad h \rightarrow-h
$$

Terms such as

$$
\mathbf{b}^{\dagger} \mathbf{b} e^{h}+\mathbf{b b}^{\dagger} e^{-h}
$$

are allowed in the presence of the modified $p-h$ symmetry [Eq. (40)], and they would create a bias towards vortices (or holes). Fortunately, as discussed above, they are not produced.

\section{Flow equations for the distribution of $h_{i}$ and $w_{i}$}

In the previous section we derived the RG rules that govern the effective tilt and hopping matrix elements. By using these $R G$ rules we can gradually decrease the energy scale of the Hamiltonian while determining the high energy parts of the ground state. When the energy scale reaches zero, the Hamiltonian that we started with is effectively diagonalized. The RG rules on their own, however, do not tell us much about the eigenvalue spectrum. To obtain useful information, we need to convert the RG rules into differential flow equations for the distributions of bond strength and tilt. As pointed out before, this has been essentially done already in the corresponding Hermitian problem.

Equation (39) provides RG rules that are identical to the case of the random $X X$ Hamiltonian. ${ }^{16}$ In this case we renormalize the bond strength and the length of bonds. Equations (39) suggest that for random vortex hopping in the nonHermitian problem, the length of a bond is replaced by the bond's tilt. This observation allows us to use essentially all results derived for the $X X$ model in Ref. 16 and apply them in this problem. In this section we review these results as they apply to the vortex-hopping problem.

We need the flow equation for the joint probability distribution $\rho(w, h)$ of the hopping strength and tilt on a bond with respect to the gradually decreasing energy scale $\Omega$. As in the theory of random spin chains, we introduce a logarithmic variable $\zeta$ to replace the bond's strength, and a renormalization-group flow parameter $\Gamma$ :

$$
\begin{gathered}
\zeta_{i}=\ln \frac{\Omega}{w_{i}}, \\
\Gamma=\ln \frac{\Omega_{0}}{\Omega},
\end{gathered}
$$

where $\Omega_{0}$ is the initial energy scale (largest matrix element) in the problem. The logarithmic energy scale $\Gamma$ is the RG flow parameter, and it increases as the energy scale is decreased. Note also that in every step of the RG we renormalize away bonds with $\zeta \rightarrow 0$. In the continuum limit, one can verify that to generate the flow $\Gamma \rightarrow \Gamma+d \Gamma$ we renormalize all bonds with $0 \leqslant \zeta<d \Gamma$. The logic behind the definition of $\zeta$ in Eq. (41) becomes clear upon considering the first RG rule in terms of the new variables. Equation (39) takes the form

$$
\zeta_{i-1, i+2}^{\text {eff }}=\zeta_{i-1}+\zeta_{i+1} \text {. }
$$

Upon transforming $\rho(w, h)$ into the new variables, we obtain the joint probability distribution $P(\zeta, h)$. The flow equation for $P(\zeta, h)$ is given by ${ }^{16}$

$$
\begin{aligned}
\frac{d P(\zeta, h)}{d \Gamma}= & \frac{\partial P(\zeta, h)}{\partial \zeta}+\iint d \zeta_{1} d \zeta_{2} d h_{0} d h_{1} d h_{2} \delta\left(\zeta-\zeta_{1}-\zeta_{2}\right) \\
& \times \delta\left(h-h_{0}-h_{1}-h_{2}\right) P\left(0, h_{0}\right) P\left(\zeta_{1}, h_{1}\right) P\left(\zeta_{2}, h_{2}\right) .
\end{aligned}
$$

Equation (43) greatly simplifies if we take the Laplace transform with respect to $h$. If $y$ is the Laplace transform variable conjugate to $h$, we obtain 


$$
\begin{aligned}
\frac{d P(\zeta, y)}{d \Gamma}= & \frac{\partial P(\zeta, y)}{\partial \zeta}+\iint d \zeta_{1} d \zeta_{2} \delta\left(\zeta-\zeta_{1}-\zeta_{2}\right) \\
& \times P(0, y) P\left(\zeta_{1}, y\right) P\left(\zeta_{2}, y\right) .
\end{aligned}
$$

The derivation of this equation is given in detail in Ref. 16.

\section{Universal fixed point distributions of $w$ and $h$, length energy scaling, and density of states}

Remarkably, Eq. (44) has a scaling solution which is an attractor to essentially all boundary conditions. ${ }^{17}$ This solution is

$$
P(\zeta, y)=\frac{1}{\Gamma+\Gamma_{0}} \exp \left\{-\sqrt{y} \operatorname{coth}\left[\sqrt{y}\left(\Gamma+\Gamma_{0}\right) \zeta\right]\right\},
$$

where $\Gamma_{0}$ is a nonuniversal constant of integration. This solution, once inverse-Laplace transformed, gives the joint probability distribution of bond strengths and effective tilt. Given some initial Hamiltonian and tilt, at sufficiently low energy scales (i.e., in the limit of large system size for the vortex problem) the distribution of effective couplings will be given by Eq. (45).

From this solution we find that the average logarithmic coupling obeys

$$
\bar{\zeta}=\Gamma+\Gamma_{0},
$$

where the overbar denotes disorder averaging. Also, Eq. (46) is obtained from the marginal distribution for the logarithmic couplings, i.e., Eq. (45) with $y \rightarrow 0$. In addition we infer that the Laplace parameter conjugate to the tilt scales as

$$
\bar{y} \sim\left(\Gamma+\Gamma_{0}\right)^{-2},
$$

and therefore the tilt field scales as

$$
\overline{h_{\mathrm{eff}}} \sim\left(\Gamma+\Gamma_{0}\right)^{2} .
$$

From the above scaling we can infer the average length of a bond after the RG reached the logarithmic energy scale $\Gamma$. This scaling is identical to the tilt-energy scaling, as mentioned above

$$
l=l_{0}\left(\Gamma+\Gamma_{0}\right)^{2} .
$$

The concomitant relation to the length-energy scaling is the relation between the fraction of unoccupied pinning sites $f$ and the energy. This relation has to be

$$
f=\frac{f_{0}}{\left(\Gamma+\Gamma_{0}\right)^{2}},
$$

where $\Gamma_{0}$ and $f_{0}$ are nonuniversal constants that depend on the particular realization of the disorder.

A formula used by Shnerb and Nelson ${ }^{11,12}$ and Brouwer et $a l .{ }^{9}$ will provide us with a connection between the spectrum of the Hermitian Hamiltonian (with zero tilt) and the spectrum of the tilted Hamiltonian, see Sec. VI B. In order to use this formula to find information about delocalized vortex states, however, we will need knowledge of the density of states in the zero-tilt limit.

The density of states (DOS) can also be derived from Eq. (49). The number of states per length in the energy interval
$\Omega \rightarrow \Omega-d \Omega$, or $\Gamma \rightarrow \Gamma+d \Gamma$ (with $\Omega=\Omega_{I} e^{-\Gamma}$ ) is just the number of bonds that get decimated at that energy scale. This, in turn, is given by

$$
f P(\zeta=0, y=0) d \Gamma .
$$

Converting this into energy terms, using Eqs. (45) and (49), we obtain

$$
g(\epsilon)=\frac{f_{0}}{\ln ^{3}\left(\Omega_{I} / \epsilon\right)} \frac{1}{\epsilon},
$$

where the energy $\epsilon$ is measured from the center of the band and

$$
\Omega_{I}=\Omega_{0} e^{\Gamma_{0}} .
$$

We expect Eq. (50) to be asymptotically exact for small $\epsilon$. The density of states, Eq. (50), exhibits the Dyson singularity. ${ }^{29}$ Similar scaling as above was obtained using alternative methods in Ref. 30.

In order to check Eqs. (46) and (49), we carried out the real-space $\mathrm{RG}$ procedure numerically on chains with 5 $\times 10^{6}$ sites for two types of distributions-the block distribution and displaced power-law distribution. In Fig. 9 we plot the average logarithmic coupling and the density of free sites vs the logarithmic flow parameter $\Gamma$ for the representatives of the two distributions types (further described in the figure caption). From these plots we also infer the values of the nonuniversal constants $f_{0}$ and $\Gamma_{0}$, which will be used later to compare results for the vortex localization problem.

\section{E. Applicability of the RSRG with nonzero tilt}

The application of the RSRG breaks down as soon as we reach energies at the mobility edge, because we can no longer write a vortex wave function which is localized between two sites-this becomes impossible as soon as the chemical potential reaches the mobility edge, and the states become extended. We can nevertheless take advantage of the real-space RG as long as we stop decimating when the energy scale reaches the mobility edge. In addition, the RSRG gives a reliable expression for the density of states of the Hamiltonian (7) with zero tilt.

\section{CONNECTION WITH VORTEX PHYSICS}

In the previous section we derived the RSRG for the vortex-hopping imaginary time Hamiltonian. In this section we will clarify the relation of the results obtained above to the physics of vortex lines with columnar pins in planar superconductors.

The vortex hopping Hamiltonian yields an energy spectrum, with filling factor determined by a chemical potential $\mu$. The RSRG outlined above is used to determine the wave function of vortices in a given chemical potential; in each step of the RG, the strongest bond is diagonalized, and if the bond energy of a vortex placed on the bond is lower than the chemical potential

$$
E_{-}=-\tilde{w}-\mu<0,
$$

then a vortex is localized on that bond. If the strongest bond in the renormalized chain is too weak to obey Eq. (52), then 

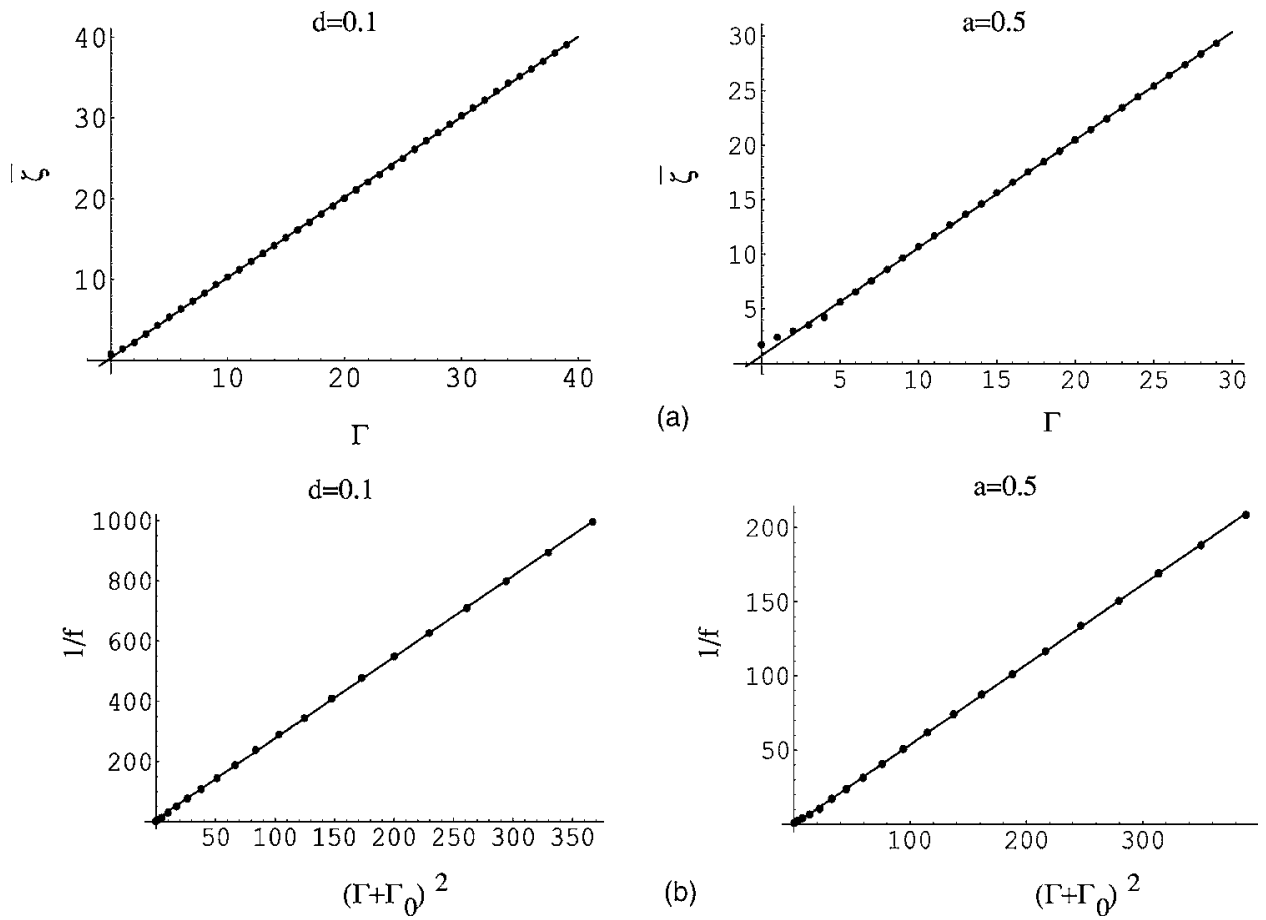

FIG. 9. Numerical real-space RG for an initial block distribution, i.e., initial hoppings uniformly distributed between $d$ and $1+d$, and for an initial "displaced power-law" distribution, with initial hoppings given by $w=0.01+w_{r}$ and $0<w_{r} \leqslant 1$ distributed as $P\left[w_{r}\right]=(1-a) \frac{1}{w_{r}^{a}}$. The latter distribution of $w_{r}$ corresponds to a hopping strength which is exponentially suppressed with the distance between pins $w_{r} \propto e^{-x}$ with $x$ distributed as $P[x] \propto e^{-(1-a) x}$. Each graph represents averaging over 20 realizations. (a) The average logarithmic coupling $\bar{\zeta}$ vs the logarithmic RG flow parameter $\Gamma$. The fitted slope in the two curves is 0.99 . From the intercept we deduce $\Gamma_{0}$. For the box distribution $\Gamma_{0}=0.31(d$ $=0.1)$ and for the power-law distribution $\Gamma_{0}=0.71(a=0.5)$. These plots should be compared with the linear dependencies predicted by Eq. (46). (b) The inverse density of free sites $1 / f$ vs $\left(\Gamma+\Gamma_{0}\right)^{2}$, with $\Gamma_{0}$ obtained from the graphs in (a). As expected from Eq. (49), the intercepts of the curves with the $y$ axis in the two plots are negligible compared to $1 / f: 2.6$ (box distribution with $d=0.1$ ) and -0.29 (power-law distribution with $a=0.5)$. From the slope of the two curves we find $f_{0}=0.37(d=0.1)$ and $f_{0}=1.85(a=0.5)$.

the RG is stopped and we have found the ground state of the zero-tilt model.

For flux lines, however, we often want to know the longitudinal magnetic field, proportional to the vortex density $n$. We need to find out how $n$ and $\mu$ are related. This is done using Eq. (49). The longitudinal vortex density vs the fraction of unoccupied sites $f$ is

$$
n=\frac{1}{2}(1-f)=\frac{1}{2}\left(1-\frac{f_{0}}{\left(\Gamma+\Gamma_{0}\right)^{2}}\right),
$$

i.e., the longitudinal vortex density is half the density of filled pinning sites, since each vortex is localized on bonds, and thus on two sites. The number of unoccupied sites $f$ as a function of energy scale is taken from Eq. (49). Now, the condition (52) gets translated to (assuming negative $\mu$; for positive $\mu$ we can carry out a particle-hole transformation)

$$
\Gamma=\ln \frac{\Omega_{0}}{|\mu|} .
$$

Upon inverting Eq. (53), we thus obtain

$$
\mu=-\Omega_{0} e^{\Gamma_{0}} \exp \left(-\sqrt{\frac{f_{0}}{1-2 n}}\right) .
$$

Note that $\Omega_{0}$ is the initial energy scale, and $f_{0}$ and $\Gamma_{0}$ are constants that are determined by the initial distribution of hoppings, and which can be calculated as in Fig. 9. Equation (55) is tested in Fig. 10 for two forms of disorder.

In the following, we will find the mobility edge, $\mu_{m}$, of the vortex-hopping model with tilt, using the RSRG. We will then use the above relations to convert the results to the physical variable $n$.

All results will depend on the initial conditions through the nonuniversal quantities $\Omega_{I}$ and $f_{0}$. Nevertheless these results are valuable since our results will be able to describe the entire phenomenology of the depinning transition in terms of these two constants, which encode the relevant part of the initial distribution. These constants can be evaluated for a given distribution either by carrying out the RSRG numerically, or by fitting curves to partial results obtained in other methods, for instance, by comparing the density of states (50) to a spectrum obtained numerically. In general, when comparing our results to spectra obtained numerically, it is easier to use formulas with $\mu$ in them rather than the physical $n$. 


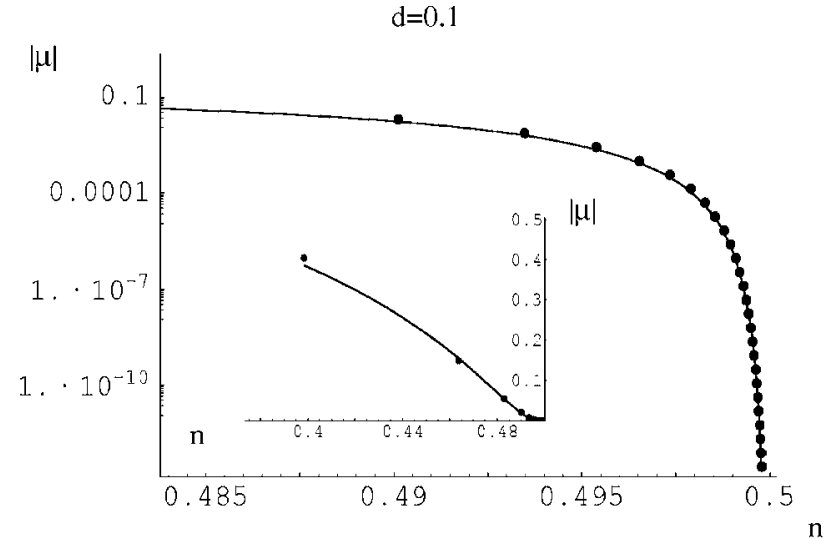

$|\mu|$

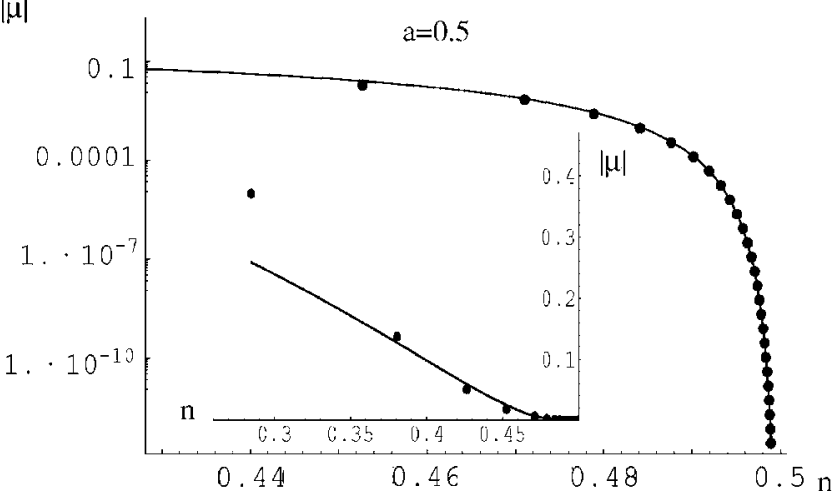

FIG. 10. Semilog plots of the vortex chemical potential $\mu$ vs the longitudinal vortex density $n$ for a box disorder distribution with $d=0.1$, and for a displaced power-law distribution with $a=0.5$, as in Fig. 9. The solid lines are Eq. (55) with $\Gamma_{0}$ and $f_{0}$ given in the caption of Fig. 9. Insets show the linear-linear plots.

\section{APPLICATION OF THE RSRG RESULTS TO NON-HERMITIAN DELOCALIZATION}

In the above we derived many characteristics of the vortex-hopping problem while assuming that all vortices are localized. The most interesting aspects of the problem, however, arise in relation to the delocalization transition of vortices with sufficient tilt.

The physical quantities of interest are essentially all related to the energy spectrum of the problem. Most particularly, given a tilt - an angle relative to the columnar defects with which we apply a magnetic field-how much magnetic field can we apply such that all vortex lines remain localized in configurations parallel to the columnar pins? This question is answered by finding the mobility edge of the nonHermitian boson-hopping model. Once we know the effective chemical potential in which the vortex bosons become depinned, the density of bosons at this chemical potential determines the critical field for destruction of the transverse Meissner effect.

Another interesting quantity is the transverse magnetic field as a function of tilt above the mobility threshold for a given longitudinal field. The transverse field is proportional to the boson current [see Eq. (8)]. For tilts slightly above the mobility threshold $h_{c}$ (i.e., a chemical potential slightly above the mobility edge), the boson current is simply given as [Eq. (A4)]:

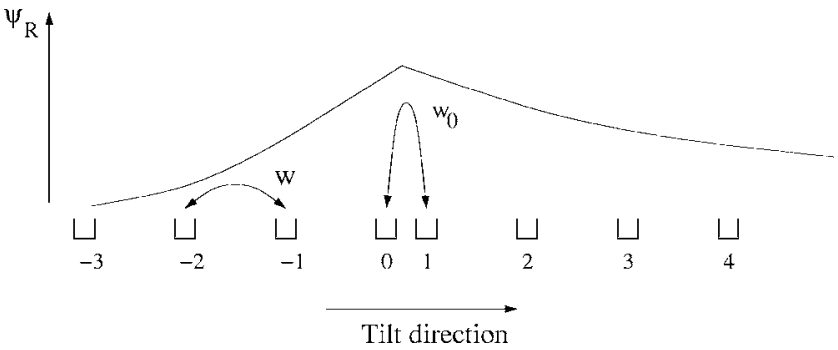

FIG. 11. Simplified model with a single strong pin. We use this model to determine the mobility edge for a given tilt. The model consists of a uniform pinning lattice with hopping $w$, periodic boundary conditions, and one pair of strongly interacting sites with hopping $w_{0}$. The strong bond localizes a vortex in it as long as the tilt is sufficiently weak. The wave function is schematically plotted, showing that its decay is asymmetric. Strong enough tilt will prevent the wave function from decaying on its right side, signaling a delocalization transition. This problem with non-hard-core vortices was considered in Ref. 10.

$$
J=\frac{1}{\pi}\left(\mu-\mu_{c}\right) \tan \theta,
$$

where $\theta$ is the angle of ascent of the spectrum in the complex energy plane (see Fig. 13). The angle $\theta$ is a universal function of the external magnetic field, or the chemical potential.

In this section we will analytically derive these quantities for the random-hopping model. First, we will find the mobility edge as a function of tilt, or, equivalently, the tilt threshold as a function of external field. In order to obtain the transverse magnetization, we will need to use a formula appearing in Refs. 9, 11, and 12, which connects the spectrum of the Hermitian boson-hopping Hamiltonian with the spectrum of the non-Hermitian problem and the tilt. We will introduce this formula, and use it to derive the quantities of interest.

\section{A. Mobility edge of the random-hopping Hamiltonian}

By using the RSRG, we demonstrated in Sec. IV that when the tilt is zero, vortices are localized between pairs of pinning sites. We also showed that as we reduce the energy scale of the Hamiltonian, and place vortices onto pairs of pinning sites that are increasingly far apart, the effective tilt $h_{\text {eff }}$ per site also increases. For any given initial tilt, at some energy scale there will no longer be a localized vortex solution. This energy is the mobility edge $\mu(h)$. Alternatively, there will be a critical longitudinal vortex density, which we denote $n(h)$, above which vortices are no longer restricted to the pinning sites. In this section we find the relation between the tilt and the critical longitudinal vortex density.

\section{Single strong bond}

We start our investigation by solving a simplified problem that will give us the correct solution. We will then proceed to justify it for the random-hopping Hamiltonian. Consider a ring of columnar defects, where there is one strong bond, $w_{0}$, and all other bonds are $w \ll w_{0}$ (Fig. 11): 


$$
\mathcal{H}=-\sum_{i \neq 0} w\left(\mathbf{b}_{i}^{\dagger} \mathbf{b}_{i+1} e^{-h}+\mathbf{b}_{i+1}^{\dagger} \mathbf{b}_{i} e^{h}\right)-w_{0}\left(\mathbf{b}_{0}^{\dagger} \mathbf{b}_{1} e^{-h}+\mathbf{b}_{1}^{\dagger} \mathbf{b}_{0} e^{h}\right) .
$$

The strong bond can localize a single vortex; we will determine at what tilt $h$ this state delocalizes.

The eigenfunctions of Hamiltonian (56) can be solved exactly. In particular, the localized wave function at the bottom of the band reads, up to normalization,

$$
|\psi\rangle=\left(\sum_{n \leqslant 0} e^{\kappa_{L}(n-1 / 2)} \mathbf{b}_{n}^{\dagger}+\sum_{n>0} e^{-\kappa_{R}(n-1 / 2)} \mathbf{b}_{n}^{\dagger}\right)|0\rangle .
$$

The left and right decay coefficients $\kappa_{L}$ and $\kappa_{R}$ are given by

$$
\begin{aligned}
& \kappa_{R} a=\ln \frac{w_{0}}{w}-h, \\
& \kappa_{L} a=\ln \frac{w_{0}}{w}+h,
\end{aligned}
$$

where $a$ is the lattice constant.

A localized solution of the form (57) can only exist as long as both $\kappa_{R}$ and $\kappa_{L}$ are positive; otherwise we cannot accommodate the periodic boundary conditions (as the system is a ring). Hence, the threshold tilt is given by the condition that $\kappa_{R}=0$ or

$$
h_{c}=\ln \frac{w_{0}}{w} .
$$

\section{Generalization for the random-hopping model}

Let us now generalize this result to the case of random hopping. At every stage of the RG we assume that the strongest bond localizes a vortex, with energy $E=\Omega=\Omega_{0} e^{-\Gamma}$. If we assume that all other bonds are the same, the problem reduces to the localizing bond problem above. However, we need a generalized condition that takes into account the randomness of the weak bonds. In this case the natural generalization of condition (59) would be

$$
h_{\mathrm{eff}}^{c}=\overline{\ln \frac{w_{0}}{w}}=\overline{\ln \frac{\Omega}{w}}=\Gamma+\Gamma_{0},
$$

where the last equality is due to Eq. (46). The reason for writing $h_{\text {eff }}$ is to remind ourselves that the tilt $h$ we consider in this section is the renormalized $h$. In the next section we connect the results derived above with the bare parameters of the vortex-pinning problem.

So far Eq. (60) is just a physically motivated guess. There are, however, several more rigorous ways to derive it. The most straightforward proof is obtained via perturbation theory. Consider the Hamiltonian (31), and assume that coupling $w_{0}$ is by far the strongest in the chain. From Eq. (34) we know what the zeroth order solution for the wave function is

$$
|-\rangle=\frac{1}{\sqrt{2}}\left(e^{-h / 2} \mathbf{b}_{0}^{\dagger}+e^{h / 2} \mathbf{b}_{1}^{\dagger}\right)|0\rangle .
$$

From perturbation theory, we can easily see that the wave function at site $|m\rangle$, to lowest nonvanishing order in the $w_{i}$ 's, reads (assume $m>0$ )

$$
\begin{aligned}
\langle m \mid-\rangle & \approx\left(\prod_{i=2}^{m} \frac{\left\langle i\left|w_{i} e^{h} \mathbf{b}_{i}^{\dagger} \mathbf{b}_{i-1}\right| i-1\right\rangle}{w_{0}}\right) \frac{\left\langle 2\left|w_{1} e^{h} \mathbf{b}_{2}^{\dagger} \mathbf{b}_{1}\right|-\right\rangle}{w_{0}} \\
& =\frac{e^{h / 2}}{\sqrt{2}} \prod_{i=1}^{m}\left(\frac{e^{h} w_{i}}{w_{0}}\right)^{m-1} .
\end{aligned}
$$

By requiring that the product at the end of Eq. (62) remains finite as $m \rightarrow \infty$, we obtain condition (60). Another derivation is supplied in Appendix B.

\section{Critical tilt and field}

As explained in Sec. IV above, the RSRG eliminates the strongest hoppings in the Hamiltonian (7) and gradually populates localized vortex states, until the energy scale of the renormalized Hamiltonian reaches the chemical potential

$$
\Omega=\max _{i}\left\{\widetilde{w}_{i}\right\}=-\mu,
$$

where the tilde indicates that the maximum is evaluated over the set of remaining (and renormalized) bond strengths. The chemical potential $\mu$ in this case is a tuning parameter that controls the vortex filling factor of the lattice, and hence the longitudinal vortex density. In terms of the RG variables $\Omega$ $=\Omega_{0} e^{-\Gamma}$ and, therefore, we can define

$$
\Gamma_{\mu}=\ln \frac{\Omega_{0}}{-\mu} .
$$

From the RG procedure, and in particular from Eq. (47), we know that

$$
\bar{h}_{\mathrm{eff}} \approx h_{0}\left(\Gamma_{\mu}+\Gamma_{0}\right)^{2} / f_{0},
$$

where $\Gamma_{0}$ and $f_{0}$ were defined in Eqs. (46) and (49), respectively, and $h_{0}$ is the unrenormalized tilt per lattice site.

In order to find a relationship between the mobility edge of the vortex-hopping problem and the unrenormalized tilt $h$, we substitute the relation $h_{\text {eff }}^{c}=h_{c}\left(\Gamma_{\mu}+\Gamma_{0}\right)^{2} / f_{0}$ into Eq. (60) and obtain

$$
h_{c}=\frac{f_{0}}{\Gamma_{\mu}+\Gamma_{0}} .
$$

The physical quantity in the vortex-pinning problem that the parameters $\mu$ and $\Gamma_{\mu}$ determine is the vortex density $n$. As a function of $\Gamma_{\mu}$, we showed in Sec. V that

$$
n=\frac{1}{2}-\frac{f_{0}}{2\left(\Gamma_{\mu}+\Gamma_{0}\right)^{2}}
$$

and, therefore, the connection between the critical tilt and the longitudinal field is 


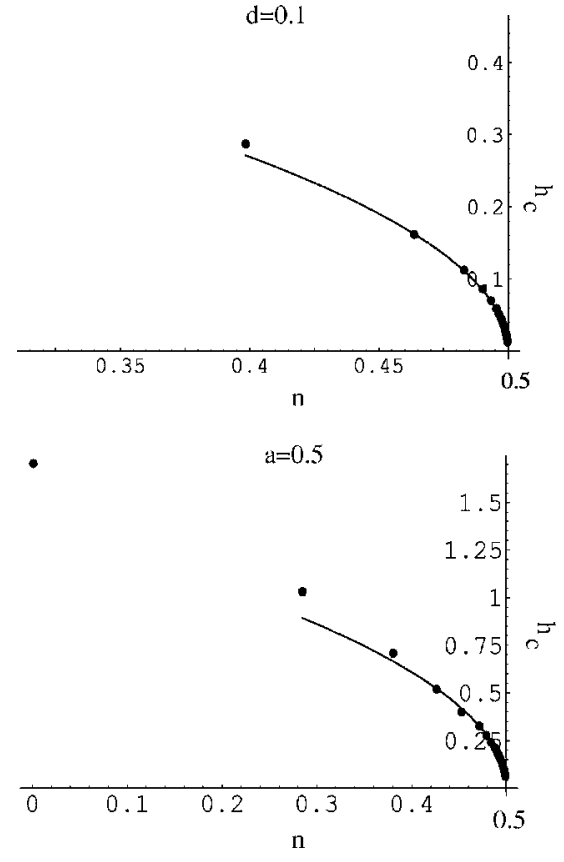

FIG. 12. Critical tilt $h_{c}$ vs longitudinal vortex density $n$ for the box distribution (top) and power-law distribution (bottom) from Fig. 9. The dots are the result of the numerical implementation of the RG, where the critical tilt is the sum of $\zeta$ divided by the full (bare) length of the chain. The solid line is a plot of Eq. (66).

$$
h_{c}=\sqrt{f_{0}(1-2 n)} \text {. }
$$

This is the main result of this section. At a given field $n$, as we increase the bare tilt, when the tilt reaches $h_{c}$, vortices get delocalized and transverse flux ensues. The critical tilt vanishes as $n \rightarrow 0.5$, i.e., as vortex states fill the lattice up to the middle of the band. This is since the localization length of the tilt-free vortex states diverges as the chemical potential approaches zero. We demonstrate the validity of Eq. (66) using the numerical real-space RG in Fig. 12. Note that the RSRG approach is most accurate near the middle of the band, where $n \sim 0.5$, in the region where the density of states diverges as in Eq. (50), also see Sec. VII.

\section{Vortex localization length}

Another quantity of interest is the vortex localization length. According to Ref. 4, this length $\xi_{\perp}$ is defined as the decay distance of the ket describing the least localized vortex in the tilt direction. From Eq. (58) and its generalization according to Eq. (60) we see that $\widetilde{\xi}_{\perp}=\frac{1}{\kappa_{R}} \approx \frac{1}{\ln \Omega / w}-h_{\text {eff }}$, where the tilde indicates that the length is a renormalized length, and not bare length. It follows that

$$
\tilde{\xi}_{\perp}=\frac{h_{c}}{\Gamma_{\mu}+\Gamma_{0}} \frac{1}{h_{c}-h} .
$$

To convert the renormalized length to physical length, we need to divide by the fraction of empty pinning sites, given by Eq. (49). In terms of physical (bare) length scales, the localization length is

$$
\xi_{\perp}=\frac{1}{h_{c}-h}
$$

\section{B. Non-Hermitian spectral formula}

A formula in Refs. 11 and 12 (see also Ref. 9) relates the imaginary part of the eigenenergies of delocalized states with the spectrum of the Hermitian-hopping problem, in the absence of a tilt. This connection, along with our detailed knowledge of the random-singlet phase, allows us to probe even the delocalized states, even though the RSRG does not strictly apply for these states. This remarkable state of affairs stems from the analytic properties for a next-neighbor hopping problem.

The non-Hermitian spectral formula is discussed extensively in Refs. 11 and 12, but we quote it here for completeness:

$$
\prod_{i=1}^{N}\left(E-\epsilon_{i}\right)=2[\cosh (h N)-1] \prod_{i=1}^{N}\left(-w_{i}\right),
$$

where $E$ is an eigenvalue of the non-Hermitian vortexhopping problem with tilt $h$ per site. There are a total of $N$ sites arranged in a ring. The $\epsilon_{i}$ 's are the eigenvalues of the zero-tilt Hermitian problem. This is a complex equation, and we will use it primarily to find a relation between the imaginary part of $E$ and the excess tilt $h-h_{c}$. In principle, we can use the above formula to find all eigenvalues of the nonHermitian problem, since we know the spectrum of the zerotilt problem from the RSRG. This strategy can be implemented numerically.

A particularly useful form of the non-Hermitian spectral formula follows. Let us assume that $N$ and $N h$ are large, and take the logarithm of Eq. (69). We obtain

$$
\sum_{i=1}^{N} \ln \left(E-\epsilon_{i}\right)=N|h|+\sum_{i=1}^{N} \ln \left(w_{i}\right)+i \pi N .
$$

In Appendix B we show how the RSRG result (60) can be deduced from the discrete non-Hermitian spectral formula. There it is also shown that we can, in fact, apply the RSRG directly to formula (70). The advantage of doing so is that in advanced stages of the RSRG the distribution of $w_{i}$ is known from the fixed point solution (45).

An important formulation of Eq. (70) is obtained by taking its real part

$$
\sum_{i=1}^{N} \frac{1}{2} \ln \left[\left(\operatorname{Re} E-\epsilon_{i}\right)^{2}+(\operatorname{Im} E)^{2}\right]=N|h|+\sum_{i=1}^{N} \ln \left(w_{i}\right) .
$$

Equation (71) specifies a curve in the complex $E$ plane on which the eigenvalues of the vortex-hopping problem lie. In the absence of the imaginary part of this equation it is impossible to determine the location of individual eigenvalues. But if we are interested in the shape of the curve, (71) is all that is necessary. In the following we will make use of the above formulas in the context of the random-hopping prob- 

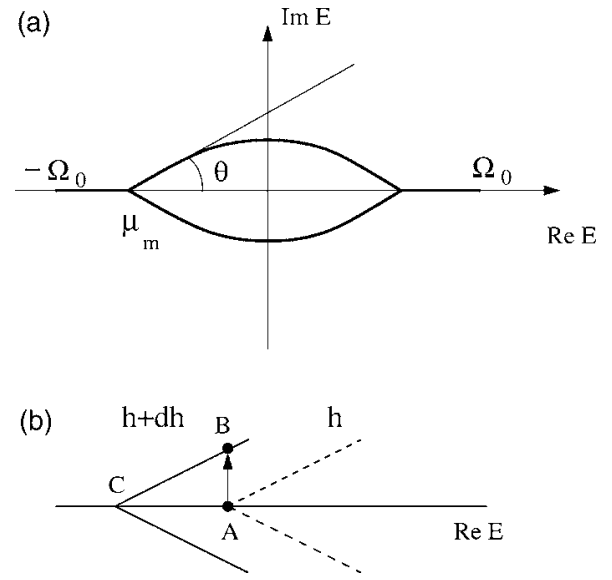

FIG. 13. (a) Typical spectrum of the random vortex-hopping Hamiltonian with nonzero tilt. The energy eigenvalues lie on the bold lines. Localized states lie on the real axis, while delocalized states have an imaginary component. $\Omega_{0}$ is the strongest bond in the bare Hamiltonian, and is of the order of $\Omega_{I}$, which is the parameter used to define $\Gamma$, the logarithmic energy scale. $\theta$ is the angle of approach of the complex branch of the spectrum, and is a critical property of the depinning transition. (b) To calculate $\theta$ we concentrate on the wedge at the mobility edge. The bold line is the spectrum at tilt $h+d h$ and the dashed is the complex spectrum at tilt $h$.

lem to obtain analytical expressions that describe the depinning transition and the spectrum of the delocalized states.

\section{Angle of approach at the mobility edge}

An interesting quantity which enters the critical properties of the depinning transition is the angle of approach of the delocalized branch of the spectrum relative to the real axis, as shown in Fig 13. This quantity is related to the current of delocalized "bosons" (i.e., the number of tilted vortices), as is discussed in the next section.

The calculation of the angle of approach is somewhat subtle, and we refer to Fig. 13(b) to aid the discussion. The figure shows the line on which the spectrum lies when the tilt is $h+d h$ in bold. The dashed bold lines indicate the line of spectrum for the lower tilt $h$. The angle of approach is given by the ratio of the segments: $\tan \theta=A B / A C$.

We first determine the vertical distance between points $A$ and $B$ in the figure. For this purpose we differentiate the real part of the non-Hermitian spectral formula (71) with respect to $\operatorname{Im} E$ while keeping Re $E$ constant. To avoid confusion we use a representation in terms of differences, taking the difference between the expression with $\operatorname{Im} E=c$ and $\operatorname{Im} E=c$ $+d e$. Upon passing to a continuum representation and introducing a density of states $g(\epsilon)$, we obtain

$$
\int_{-\Omega_{0}}^{\Omega_{0}} d \epsilon g(\epsilon) \frac{c \cdot d e}{\left(\operatorname{Re} E-\epsilon_{i}\right)^{2}+c^{2}}=d h .
$$

In the limit of $d h \rightarrow 0$, the integrand on the left is nothing but $g(\epsilon) d e \pi \delta_{(\operatorname{Re} E-\epsilon)}$, and we obtain (where we drop the Re sign) $\pi g(E) d e=d h$ and hence find that $A B$ in Fig. 13(b) is

$$
d e=d h \frac{|E| \ln ^{3} \frac{\Omega_{I}}{|E|}}{\pi f_{0}},
$$

where $g(E)$ is taken from Eq. (50). In Eq. (73) $E$ designates the energy of the mobility edge, so we substitute

$$
E=\mu_{m}(h) \text {. }
$$

The second part of the calculation focuses on $A C$. This is the distance the mobility edge travels as the tilt changes from $h$ to $h+d h$. But from Eq. (65) we can find this quantity directly:

$$
\frac{d h}{d \mu_{m}}=\frac{f_{0}}{|\mu|\left(\Gamma_{\mu}+\Gamma_{0}\right)^{2}}=\frac{f_{0}}{\left|\mu_{m}\right| \ln ^{2} \frac{\Omega_{I}}{|\mu|}}
$$

The angle of approach is now readily found:

$$
\tan \theta=\frac{d e}{d \mu_{m}}=\frac{d h}{d \mu_{m}} \frac{d e}{d h}=\frac{\Gamma+\Gamma_{0}}{\pi}=\frac{1}{\pi} \ln \frac{\Omega_{I}}{\left|\mu_{m}\right|} .
$$

Again it is a universal form, which depends on the initial distribution only through a single parameter $\Omega_{I}$. Note that as the tilt $h$ approaches zero, and the mobility edge approaches the "middle of the band" (i.e., $\mu_{m} \rightarrow 0$ ), the angle $\theta$ approaches $\pi / 2$.

\section{Vortex current near the mobility edge}

The imaginary vortex current near the onset is in general given by Eq. (A4) in Appendix A

$$
J_{\text {total }}=2 \frac{1}{L} \sum_{k_{n}} \operatorname{Re} \frac{\partial E_{n}}{\partial h}=\frac{1}{\pi}\left(\operatorname{Im} E_{\mu}-\operatorname{Im} E_{\mu_{m}}\right),
$$

where $L$ is the number of sites in the lattice. In particular, we can apply this formula to the random-hopping problem. In Eq. (75) the angle of approach near the delocalization transition was found (also see Fig. 13):

$$
\frac{d \operatorname{Im} E}{d \epsilon}=\tan \theta=\frac{1}{\pi} \ln \frac{\Omega_{I}}{\left|\mu_{m}\right|},
$$

where we define $\epsilon$ as

$$
\epsilon=\operatorname{Re} E,
$$

and $\mu_{m}$ is the chemical potential at the mobility edge. Now, near the onset of transverse flux penetration we can write

$$
\begin{aligned}
J_{\text {total }} & =\frac{1}{\pi}\left(\operatorname{Im} E_{\mu}-\operatorname{Im} E_{\mu_{m}}\right) \approx \frac{1}{\pi}\left(\mu-\mu_{m}\right) \tan \theta \\
& =\frac{1}{\pi^{2}} \ln \frac{\Omega_{I}}{\left|\mu_{m}\right|}\left(\mu-\mu_{m}\right) .
\end{aligned}
$$

This result can also be expressed in terms of the difference between the longitudinal vortex density and its critical value. The number of extra vortices per length is just $n-n_{c}$. Therefore the transverse vortex density, i.e., the imaginary "boson" current, is also 


$$
J_{\text {total }} \approx \frac{1}{L} \operatorname{Im} \frac{\partial \epsilon}{\partial k}\left(n-n_{c}\right) \approx\left(n-n_{c}\right) \frac{\tan \theta}{\pi g\left(\mu_{m}\right)} .
$$

Close to $n_{c}$, we may assume that the real parts of the energy of eigenstates near the mobility edge do not change as they undergo the delocalization transition. Therefore we can use Eq. (50) for the DOS. Upon combining Eq. (50) with Eqs. (55), (65), and (66) we can write result (78) in terms of the longitudinal vortex density only:

$$
J_{\text {total }} \approx\left(n-n_{c}\right) \frac{\Omega_{I} f_{0}}{\pi^{2}} \frac{1}{(1-2 n)^{2}} e^{-\sqrt{f_{0} /(1-2 n)}}
$$

or in terms of the tilt only:

$$
J_{\text {total }} \approx\left(h-h_{c}\right) \frac{\Omega_{I} f_{0}^{2}}{\pi^{2}} \frac{1}{h^{3}} e^{-f_{0} / h} .
$$

Equations (79) and (80) are the main results of this section.

One can actually use the above reasoning to obtain not only the total current, but the current of individual states near the mobility edge. Consider the state $E_{n}$ which can be associated with a wave vector $k_{n}$. As we change $k=k_{n}$ to $k_{n}+\frac{2 \pi}{L}$, we move from the eigenvalue $E_{n}$ to $E_{n+1}$. Thus

$$
\operatorname{Re}(d E)=\epsilon_{n+1}-\epsilon_{n} \approx \frac{2}{\operatorname{Lg}(\epsilon)},
$$

where we make use of Eq. (50), and again set $\epsilon=\operatorname{Re}(E)$. The factor 2 in the numerator of Eq. (A3) is due to the real spectrum splitting into two branches, and we choose a convention in which both branches of the spectrum contribute to the DOS. Now we can also substitute $d k=\frac{2 \pi}{L}$, and we obtain for the current of this state

$$
J_{n}=\frac{1}{L} \operatorname{Re} \frac{\partial E_{n}}{\partial h}=\frac{1}{L} \operatorname{Im} \frac{\partial E}{\partial k} \approx \frac{2}{L g(\epsilon)} \frac{1}{2 \pi} \tan \theta=\frac{\tan \theta}{L \pi g(\epsilon)},
$$

where we used $\tan \theta=\frac{d \operatorname{Im} E}{d \epsilon}$. The result (82) can be written in terms of the longitudinal vortex density $n$ instead of in terms of $\epsilon$ :

$$
J_{n} \approx \frac{f_{0} \Omega_{I}}{L \pi^{2}} \frac{1}{(1-2 n)^{2}} e^{-\sqrt{f_{0} /(1-2 n)}} .
$$

Equation (82) can also be expressed in terms of the tilt $h$, using Eq. (66):

$$
J_{n} \approx \frac{f_{0}^{3} \Omega_{I}}{L \pi^{2}} \frac{1}{h^{4}} e^{-f_{0} / h}
$$

\section{EXACT DIAGONALIZATION OF FINITE SYSTEMS}

In this section we analyze the free-fermion Hamiltonian (13) by exact numerical diagonalization of finite-size systems with up to $N=4000$ lattice sites. While this study is mostly focused on the case of random hopping, we also present some results for on-site disorder. We employ periodic boundary conditions in order to model qualitatively a realis-

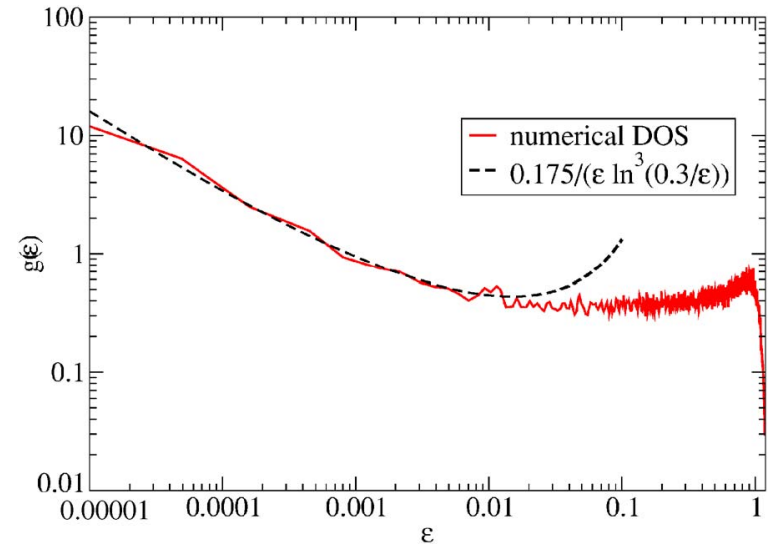

FIG. 14. (Color online) Density of states of the random-hopping problem with zero tilt for 2000 lattice sites, averaged over 50 disorder realizations. The hopping parameters $w_{i}$ are equally distributed in $[0.2,0.7]$. At low energies, good agreement is obtained with the analytic RSRG result Eq. (50).

tic superconducting slab where vortices can enter and exit at the edges. Note that for open boundary conditions (i.e., zero hopping matrix elements at the edges), the magnetic field tilt could be gauged away and would not induce any transverse vortex density. Since all quantities are random, we average over a finite number of disorder realizations $N_{R}=20 \cdots 100$. Unless indicated otherwise, the random-hopping matrix elements $w_{i}$ are drawn from a flat distribution in $[c, c+0.5]$, where the lower cutoff $c=0.2$ is used to prevent singular couplings close to zero, which would lead to almost decoupled subsystems. For diagonal disorder, we use on-site energies $\epsilon_{i}$ which are evenly distributed in $[-0.5,0.5]$.

We first study the case of vanishing tilt $h=0$, in order to illustrate the qualitatively different physics of bond and onsite disorder. For bond disorder, we have calculated the density of states from the real part of the single-particle spectrum, averaging over $N_{R}=50$ realizations of the disorder. The results are shown in Fig. 14, where it is evident that the DOS diverges at the center of the band, as predicted by the RSRG. We obtain a good fit to the theoretical result in Eq. (50).

A similar behavior is obtained for the localization length $\xi$, which we define as

$$
\xi=\sum_{i, j=1}^{L}\left|\Psi_{i}\right|^{2}\left|\Psi_{j}\right|^{2} d_{c}(i, j),
$$

where

$$
d_{c}(i, j)=\frac{L}{\pi} \sin \left(\frac{\pi|i-j|}{L}\right)
$$

is the chord distance on the ring and $\Psi_{i}$ is the wave function at site $i$ of the single-particle state under consideration. Results are shown in Fig. 15. For random hopping we find that the localization length diverges in the middle of the band according to 

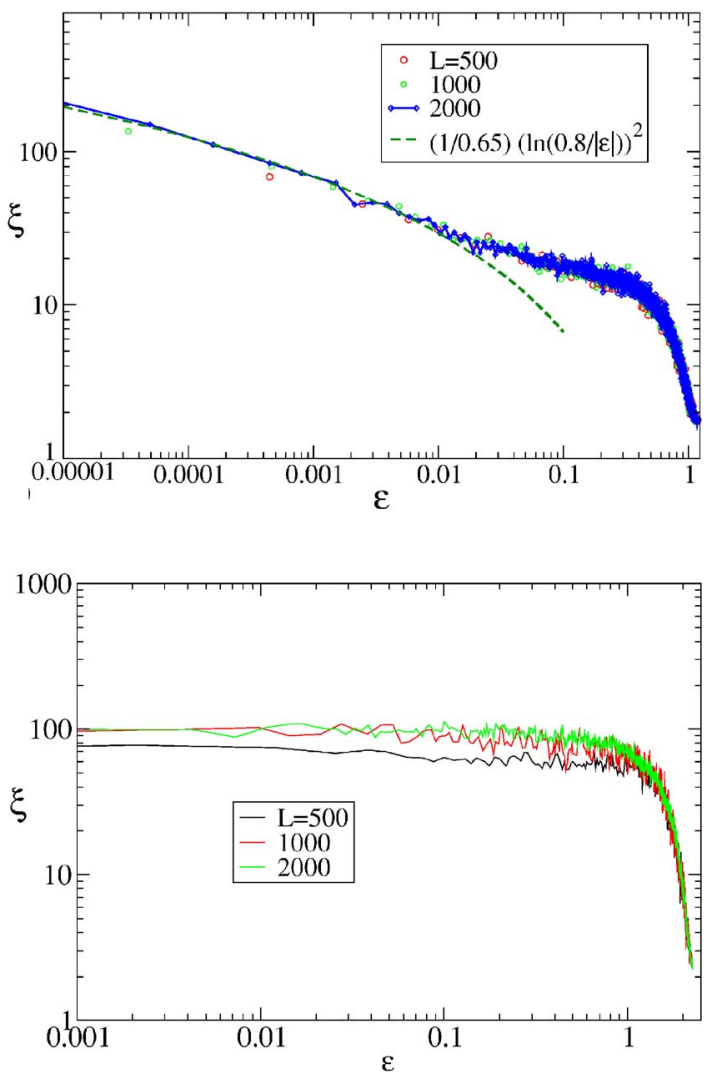

FIG. 15. (Color online) Top: localization length of the randomhopping problem with zero tilt for different system sizes $L$, averaged over 50 disorder realizations. As in Fig. 14, the hopping matrix elements $w_{i}$ are equally distributed in $[0.2,0.7]$. The low-energy behavior agrees very well with the result $\xi \sim \frac{1}{f_{0}} \ln ^{2}\left(\Omega_{I} /|\epsilon|\right)$ predicted by the RSRG. Bottom: localization length for on-site disorder with site energies $\epsilon_{i}$ equally distributed in $[-0.5,0.5]$, averaged over 10 realizations. $\xi(\epsilon)$ clearly stays finite as $\epsilon \rightarrow 0$.

$$
\xi=l \sim \frac{1}{f_{0}} \ln ^{2} \frac{\Omega_{I}}{|\epsilon|}
$$

as predicted by the RSRG [Eq. (48)]. In particular, there is always a vortex state at zero energy which is delocalized across the whole system. On the other hand, for on-site disorder $\xi$ clearly stays finite, as shown in the right graph of Fig. 15. As a result, for bond disorder an arbitrarily small but finite tilt $h$ leads to extended states at the center of the band, in contrast to the case of on-site disorder where this only happens above a critical tilt $h>h_{c}{ }^{4}$ (Note that the localization length discussed here is the distance between the two pinning sites that share a vortex, as opposed to $\xi_{\perp}$ of Sec. VI A, which describes the wondering of the vortex away from the pinning sites due to the tilt.)

We now present results for finite tilt $h>0$. In this case the Hamiltonian is non-Hermitian, with complex eigenvalues and left/right eigenstates which are no longer equal. In Fig. 16 we show numerical results for the spectrum of the random-hopping problem. For small tilt almost all states are localized and the corresponding eigenenergies real. As the tilt increases, the two mobility edges $\pm \mu_{c}$ move towards the
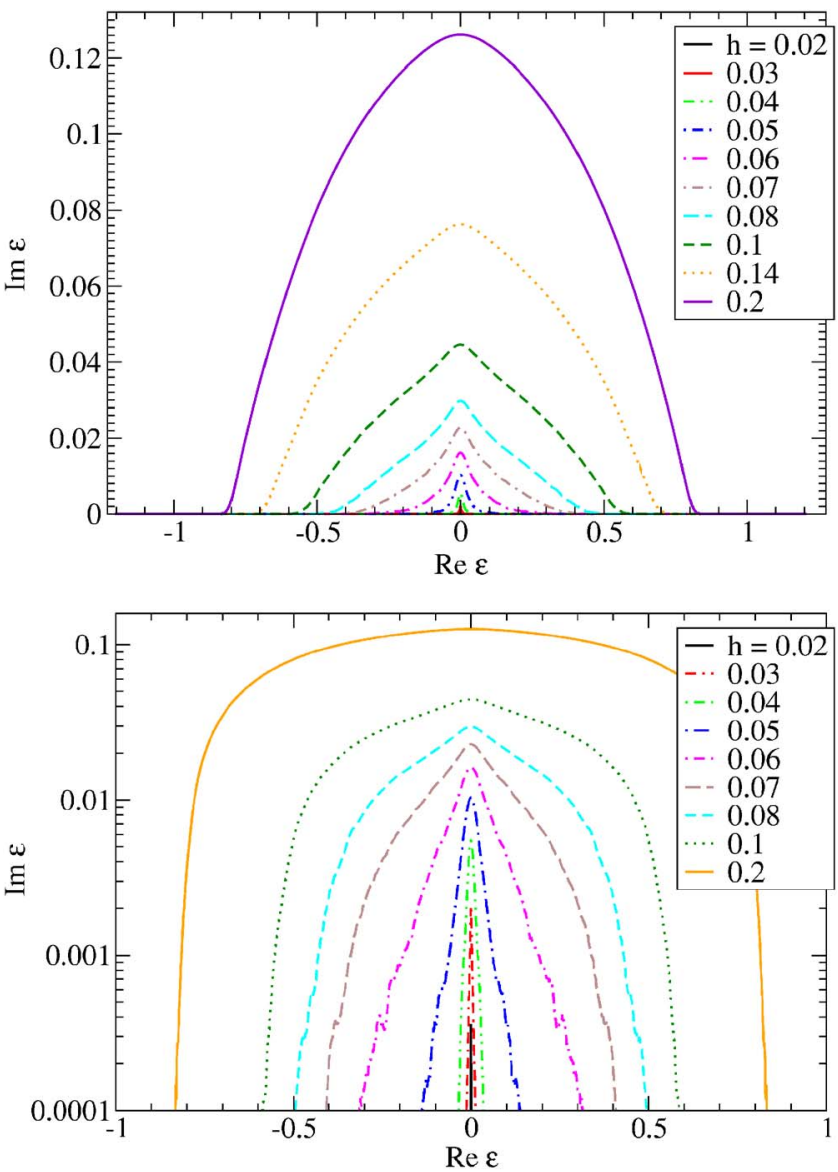

FIG. 16. (Color online) Spectrum of single-particle states for different values of the tilt, with hopping disorder as in Fig. 14, $L$ $=2000$ lattice sites and averaging over 100 disorder realizations, on a linear scale (top) and in a lin-log plot (bottom).

band edges and define a growing region of extended states. In Fig. 17, the dependence of $\mu_{c}$ on the tilt is shown. The rapid vanishing of $\mu_{c}$ at small $h$ is consistent with Eq. (55) using Eq. (66) (two fits are shown), although a quantitative comparison is difficult due to the finite system size.

At finite tilt, the most interesting physical quantity for the vortex problem is the transverse flux, corresponding to the

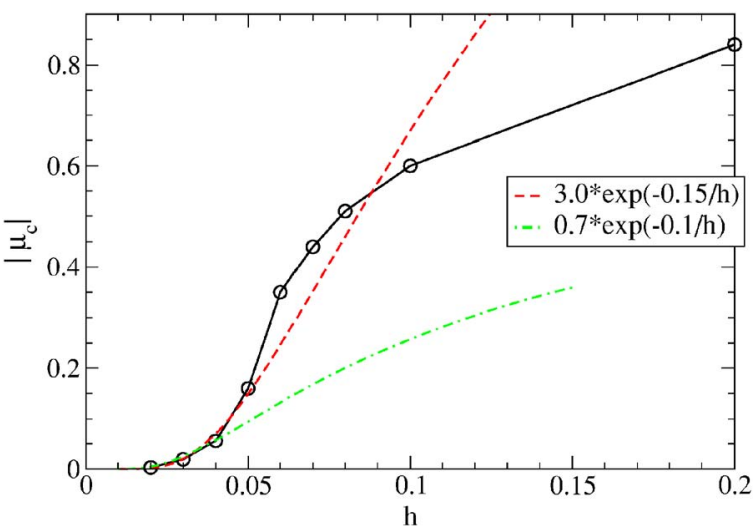

FIG. 17. (Color online) Mobility edge vs tilt for different values of the tilt, with hopping disorder as in Fig. 14, $L=2000$ lattice sites, and averaging over 100 disorder realizations. 


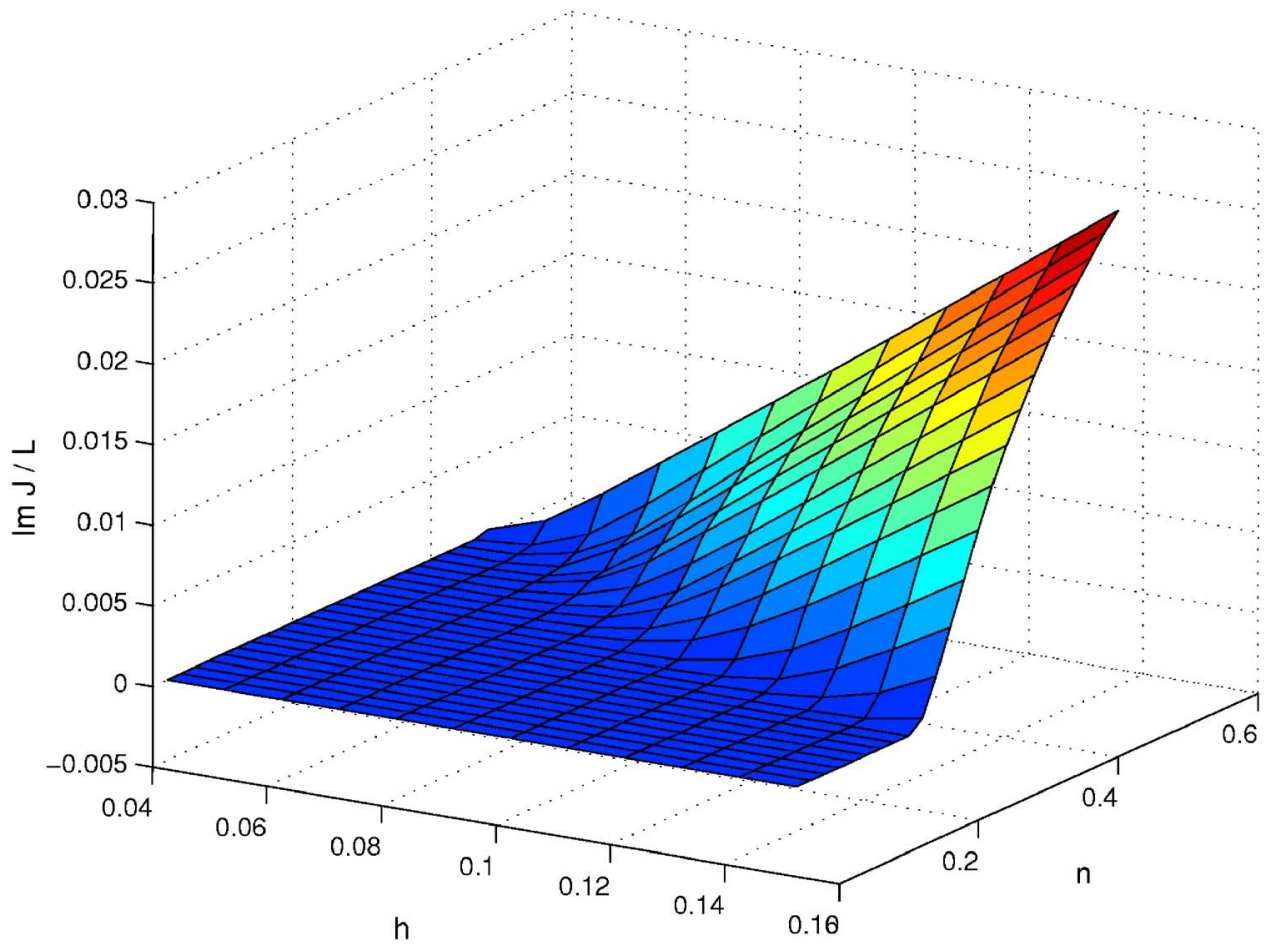

FIG. 18. (Color online) Imaginary current for random hopping distributed as in Fig. 14, as a function of tilt $h$ and vortex density $n$. Results are given for $L=1000$ sites, with a disorder average performed over 20 realizations. imaginary current $J$. Following Eq. (14), we obtain the current as the derivative of the ground-state energy $E_{g}$ with respect to tilt

$$
J=(-i) \frac{1}{L} \frac{d E}{d h} .
$$

The resulting current for hopping disorder, as a function of particle (vortex) density $n$ and tilt $h$, is shown in Fig. 18. Clearly, for weak tilt and few vortices, the current vanishes since the mobility edge is close to the center of the band and all the occupied states are localized. This is a manifestation of the transverse Meissner effect: In the presence of disorder due to columnar pins, a weak transverse magnetic field does not induce a transverse magnetic flux, because the flux lines are pinned by the defects. ${ }^{3}$ With increasing filling and tilt, a well-defined transition to finite current occurs when the mobility edge $\mu_{c}$ coincides with the vortex chemical potential $\mu$. This is shown in more detail in Fig. 19, where the current is given as a function of the tilt for quarter filling $n=0.25$. Apart from finite-size rounding of the transition, the onset of the current is linear in $h$, consistent with the theoretical prediction in Eq. (80).

\section{SUMMARY}

In this paper we studied the physics of interacting vortices in a two-dimensional type-II superconductor with parallel random columnar defects in the plane. Our analysis is based on mapping the system onto a $(1+1)$-dimensional ensemble of hard-core bosons with on-site or hopping disorder. The equivalence of hard-core bosons and fermions in one spatial dimension allowed us to focus on an effectively noninteracting, but disordered, many-fermion system for the special case of Luttinger liquid parameter $g=1$. As a qualitative benchmark, we considered the exactly solvable Lloyd model with on-site disorder where we obtained analytic predictions for the critical tilt and the transverse magnetic flux above the critical tilt. We then studied extensively the case of general random hopping, using the real-space renormalization group (RSRG) technique, which we generalized to the case of finite tilt. The RSRG allowed us to completely describe the localized phase and to extract physical quantities such as the density of states or the mobility edge as a function of tilt. With the help of a non-Hermitian spectral formula we were able to extend the RSRG results to the delocalized states, and to calculate the onset of transverse magnetic flux close to the breakdown of the transverse Meissner effect, which is the most relevant experimental observable. Finally, we have

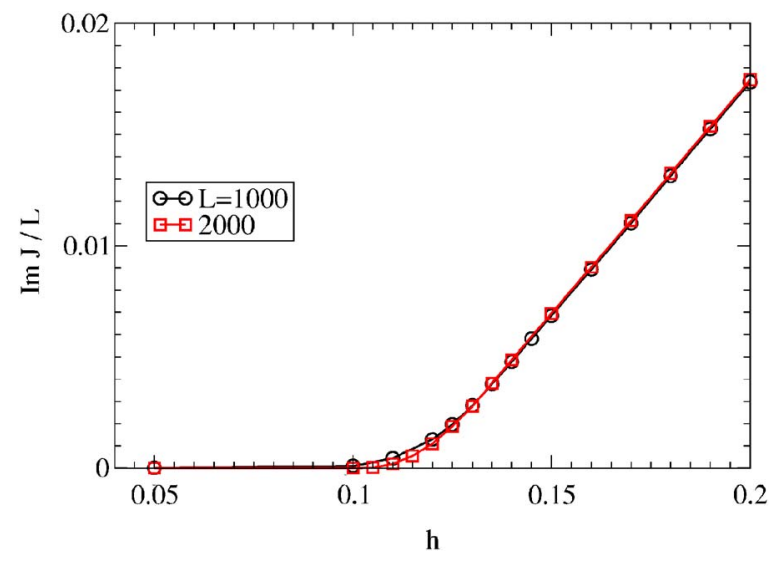

FIG. 19. (Color online) Onset of imaginary current (i.e., transverse magnetic flux) as a function of tilt, for random hopping as in Fig. 14 and vortex density $n=0.25$. Data are averaged over 20 realizations of disorder. Apart from finite-size rounding the onset is linear, as predicted by Eq. (80). 
compared our analytic predictions to numerics on finite systems which we diagonalized exactly. We found good agreement with the RSRG results, although the numerics indicates that the universal regime is only accessible for very large system sizes.

Our results seem to indicate that scaling pictures formerly held regarding the case of uniform pins at a random separation are not valid near critical tilting, particularly, we find $B_{\perp} \propto\left(H_{\perp}-H_{\perp}^{c}\right)^{\zeta}$ in $1+1$ dimensions, but with $\zeta=1$ rather than the expected $\zeta=1 / 2$ (see Secs. I and VID). We hope that our results will encourage further experimental and theoretical research on vortex pinning, both to verify our predictions, and to explore how strong randomness physics may appear in situations not touched upon here.

\section{ACKNOWLEDGMENTS}

D.R.N. would like to thank I. Affleck for discussions on torque measurements. D.R.N. was supported by the National Science Foundation through Grant No. DMR-0231631 and through the Harvard Materials Research Science and Engineering Laboratory via Grant No. DMR-0213805. G.R. would like to thank the generous hospitality of the Kavli Institute of Theoretical Physics, UCSB, and of the Boston University visitors program.

\section{APPENDIX A: A GENERAL FORMULA FOR THE TRANSVERSE VORTEX DENSITY NEAR THE MOBILITY EDGE}

If we increase the magnetic field above the critical field, some vortices are delocalized, and a transverse vortex density appears. The transverse vortex density (imaginary current) for a single vortex state is given by

$$
J_{n}=\frac{1}{L} \operatorname{Re} \frac{\partial E_{n}}{\partial h} .
$$

Following Refs. 11 and 12, we write the eigenvalues of the random-hopping problem as

$$
\epsilon_{n}(h)=E\left(h+i k_{n}\right) .
$$

The function $E(h+i k)$ is analytic and therefore obeys the Cauchy-Riemann equations. In particular,

$$
\operatorname{Re} \frac{\partial E}{\partial h}=\operatorname{Im} \frac{\partial E}{\partial k} .
$$

From Eq. (A3) we can get a general result for the total current

$$
\begin{aligned}
J_{\text {total }} & =2 \frac{1}{L} \sum_{k_{n}} \operatorname{Re} \frac{\partial E_{n}}{\partial h} \rightarrow 2 \int_{k_{\min }}^{k_{\max }} \frac{d k}{2 \pi} \operatorname{Im} \frac{\partial \epsilon}{\partial k} \\
& =\frac{1}{\pi}\left(\operatorname{Im} E_{k_{\max }}-\operatorname{Im} E_{k_{\min }}\right)=\frac{1}{\pi}\left(\operatorname{Im} E_{\mu}-\operatorname{Im} E_{\mu_{m}}\right) .
\end{aligned}
$$

From Eq. (A4) we can immediately conclude, for instance, that closed bubbles of tilted states do not contribute to the total current. The factor of 2 accounts for the two branches of the spectrum.

\section{APPENDIX B: THE RSRG AND CRITICAL TILT FROM THE DISCRETE NON-HERMITIAN SPECTRAL FORMULA}

The discrete non-Hermitian formula allows an alternative formulation of the real-space RG, and thus gives further support to the results obtained in Sec. VI. We will use the real part of the non-Hermitian spectral formula (70):

$$
\sum_{i=1}^{N} \frac{1}{2} \ln \left[\left(\operatorname{Re} E-\epsilon_{i}\right)^{2}+(\operatorname{Im} E)^{2}\right]=N|h|+\sum_{i=1}^{N} \ln \left(w_{i}\right) .
$$

Because of particle-hole symmetry, we can also write

$$
\begin{aligned}
\sum_{i=1}^{N / 2} & \frac{1}{2}\left\{\ln \left[\left(\operatorname{Re} E-\epsilon_{i}\right)^{2}+(\operatorname{Im} E)^{2}\right]+\ln \left[\left(\operatorname{Re} E+\epsilon_{i}\right)^{2}+(\operatorname{Im} E)^{2}\right]\right\} \\
& =N|h|+\sum_{i=1}^{N} \ln \left(w_{i}\right) .
\end{aligned}
$$

The real-space RG is obtained as follows. Assuming strong disorder, as we did in Sec. IV, we know that the eigenvalues $\pm \epsilon_{1}$ are associated with the strongest bond of the chain $n_{1}$ :

$$
\epsilon_{1} \approx w_{n_{1}} \text {. }
$$

Let us choose an energy $|E| \ll \epsilon_{1}$, and assume that it belongs to a localized state. We can now write

$$
\sum_{i=2}^{N / 2} \ln \left|E^{2}-\epsilon_{i}^{2}\right|=N|h|+\sum_{i=1}^{N} \ln \left(w_{i}\right)-\ln \epsilon_{i}^{2} .
$$

However, we can rearrange terms on the right-hand side as follows:

$$
\begin{aligned}
& \ln w_{n_{1}-1}+\ln w_{n_{1}}+\ln w_{n_{1}+1}-\ln \epsilon_{i}^{2} \\
& \approx \ln \left(\frac{w_{n_{1}-1} w_{n_{1}+1}}{w_{n_{1}}}\right)=\ln w_{n_{1}-1, n_{1}+2}^{\text {eff }},
\end{aligned}
$$

where $w_{n_{1}-1, n_{1}+2}^{\text {eff }}$ is just the effective hopping according to the real-space RG as found in Eq. (39). We now have the renormalized chain with the two sites $n_{1}, n_{1}+1$ removed, and we can write the spectral formula as

$$
\sum_{i=2}^{N / 2} \ln \left|E^{2}-\epsilon_{i}^{2}\right|=N|h|+\sum_{i=1}^{N-2} \ln \left(\widetilde{w}_{i}\right)-\ln \epsilon_{i}^{2},
$$

where the $\widetilde{w}_{i}$ are the hoppings of the renormalized chain. This process can now be repeated-starting with the next eigenvalue $\epsilon_{2}$ of the largest renormalized bond $\max \left\{\widetilde{w}_{i}\right\}$ $=\widetilde{w}_{n_{2}}$, the bond $n_{2}$ can be renormalized in the same way as written above. Thus we recover the real-space RG-we can eliminate the high energy states and write the spectral formula in terms of the new couplings and the reduced length. 
Note that the tilt part remains constant in the process. This invariance corresponds to the second condition in Eq. (39), which can be written as

$$
\sum_{i} h_{i}=\sum_{\tilde{i}} \tilde{h_{i}}
$$

We can repeat this process until we reach energy eigenvalues of the same order as $E$.

We can also rederive the critical tilt result (60). We assume that when close to the critical tilt, or to the mobility edge, the eigenvalue $E$, which we assume is $\epsilon_{m}<|E|<\epsilon_{m+1}$, is as far from $\epsilon_{m}, \epsilon_{m+1}$ as it can be [such that the LHS of Eq. (B6) is maximized], and therefore

$$
\frac{1}{N} \sum_{i \sim m} \ln || E\left|-\epsilon_{i}\right| \ll 1
$$

where $i \sim m$ indicates summing over $i$ 's in the vicinity of $m$ and $m+1$. Equation (B8) is justified by the above assumption and our understanding that in the random singlet phase, when renormalizing a bond with energy $E$, there is negligible probability to find another bond of similar strength next to it. With Eq. (B8), we can now write the spectral formula assuming we can carry out the RG procedure above until we arrive at the energy scale $|E|$. Also, since we ignore eigenvalues of the order of $|E|$, all remaining eigenvalues are much smaller than $|E|$. Therefore we can write

$$
\sum_{i=1}^{\tilde{N} / 2} \ln \left|E^{2}\right| \approx N|h|+\sum_{i=1}^{\tilde{N}} \ln \left(\widetilde{w}_{i}\right),
$$

where $\tilde{N}$ is the length of the chain renormalized to energy scale $|E|$. This, in turn, can be written as

$$
\sum_{i=1}^{\tilde{N}} \ln \frac{|E|}{\widetilde{w}_{i}}=\tilde{N}\left(\Gamma_{E}+\Gamma_{0}\right) \approx N h_{c},
$$

where we used Eq. (46) to express the average of the logarithmic couplings in terms of $\Gamma_{E}$. We have now made the replacement $|h| \rightarrow h_{c}$ the critical tilt. Before, we assumed that the tilt is as close to critical as possible, and therefore the left-hand side of Eq. (B10) is the maximum possible value for $N|h|$, without letting $E$ have an imaginary part. To get $|h|<h_{c}$ we only need to allow $E$ to come arbitrarily close to $\epsilon_{m}$. Thus we conclude

$$
h_{c}=\frac{\tilde{N}\left(\Gamma_{E}+\Gamma_{0}\right)}{N}=\frac{f_{0}}{\Gamma_{\mu}+\Gamma_{0}},
$$

where we used Eq. (49) for the effective density of the renormalized chain. This concludes an independent demonstration of Eq. (60).
${ }^{1}$ A. I. Larkin and Y. N. Ovchinnikov, J. Low Temp. Phys. 34, 409 (1979).

${ }^{2}$ M. V. Feigel'man, V. B. Geshkenbein, A. I. Larkin, and V. M. Vinokur, Phys. Rev. Lett. 63, 2303 (1989).

${ }^{3}$ D. R. Nelson and V. M. Vinokur, Phys. Rev. B 48, 13060 (1993).

${ }^{4}$ N. Hatano and D. R. Nelson, Phys. Rev. B 56, 8651 (1997).

${ }^{5}$ W. Hofstetter, I. Affleck, D. R. Nelson, and U. Schollwoeck, Europhys. Lett. 66, 178 (2004).

${ }^{6}$ I. Affleck, W. Hofstetter, D. R. Nelson, and U. Schollwoeck, J. Stat. Mech.: Theory Exp. (2004) P10003.

${ }^{7}$ L. Radzihovsky, Phys. Rev. B 73, 104504 (2006).

${ }^{8}$ P. Lloyd, J. Phys. C 2, 1717 (1969).

${ }^{9}$ P. W. Brouwer, P. G. Silvestrov, and C. W. J. Beenakker, Phys. Rev. B 56, R4333 (1997).

${ }^{10}$ C. Mudry, P. W. Brouwer, B. I. Halperin, V. Gurarie, and A. Zee, Phys. Rev. B 58, 13539 (1998).

${ }^{11}$ N. M. Shnerb and D. R. Nelson, Phys. Rev. Lett. 80, 5172 (1998).

${ }^{12}$ K. A. Dahmen, D. R. Nelson, and N. M. Shnerb, in The Statistical Mechanics of Biocomplexity, Proceedings of the XV Sitges Conference, edited by D. Reguera, J. M. G. Vilar, and J. M. Rubi (Springer, Berlin, 1999).

${ }^{13}$ I. Y. Goldsheid and B. A. Khoruzhenko, Phys. Rev. Lett. 80, 2897 (1998).

${ }^{14}$ S. K. Ma, C. Dasgupta, and C. K. Hu, Phys. Rev. Lett. 43, 1434 (1979).
${ }^{15}$ C. Dasgupta and S. K. Ma, Phys. Rev. B 22, 1305 (1980).

${ }^{16}$ D. S. Fisher, Phys. Rev. B 50, 3799 (1994).

${ }^{17}$ D. S. Fisher, Phys. Rev. B 51, 6411 (1995).

${ }^{18}$ K. Damle, O. Motrunich, and D. A. Huse, Phys. Rev. Lett. 84, 3434 (2000).

${ }^{19}$ E. Altman, Y. Kafri, A. Polkovnikov, and G. Refael, Phys. Rev. Lett. 93, 150402 (2004).

${ }^{20}$ F. Igloi and C. Monthus, Phys. Rep. 412, 277 (2005).

${ }^{21}$ T. P. Eggarter and R. Riedinger, Phys. Rev. B 18, 569 (1978).

${ }^{22}$ T. Hwa, D. R. Nelson, and V. M. Vinokur, Phys. Rev. B 48, 1167 (1993).

${ }^{23}$ W. Jiang, N.-C. Yeh, D. S. Reed, U. Kriplani, D. A. Beam, M. Konczykowski, T. A. Tombrello, and F. Holtzberg, Phys. Rev. Lett. 72, 550 (1994).

${ }^{24}$ S. A. Grigera, E. Morre, E. Osquiguil, C. Balseiro, G. Nieva, and F. de la Cruz, Phys. Rev. Lett. 81, 2348 (1998).

${ }^{25}$ V. Ta Phuoc, E. Olive, R. De Sousa, A. Ruyter, L. Ammor, and J. C. Soret, Phys. Rev. Lett. 88, 187002 (2002).

${ }^{26}$ R. J. Olsson, W.-K. Kwok, L. M. Paulius, A. M. Petrean, D. J. Hofman, and G. W. Crabtree, Phys. Rev. B 65, 104520 (2002).

${ }^{27}$ T. Holstein and H. Primakoff, Phys. Rev. 58, 1098 (1940).

${ }^{28}$ P. Jordan and E. Wigner, Z. Phys. 47, 631 (1928).

${ }^{29}$ F. J. Dyson, Phys. Rev. 92, 1331 (1953).

${ }^{30}$ A. D. Stone and J. D. Joannopoulos, Phys. Rev. B 24, 3592 (1981). 